\title{
Zur Kenntnis der thermischen Umlagerung des Bicyclo[1.1.0]butan-Systems. Eine kinetische Studie des Übergangs von Tricyclo[4.1.0.0 $\left.{ }^{2,7}\right]$ heptanen in Bicyclo[3.2.0]hept-6-ene
}

\author{
Manfred Christl ${ }^{* 1 \mid}$, Roland Stangl ${ }^{|2|}$ und Hans Jelinek-Fink ${ }^{|3|}$ \\ Institut für Organische Chemie der Universität Würzburg, \\ Am Hubland, D-8700 Würzburg
}

Eingegangen am 21. September 1991

Key Words: Bicyclo[1.1.0]butanes, rearrangement / Tricyclo[4.1.0.0.7.7heptanes, kinetics of thermolysis / 1,2,3-Methenonaphthalenes, 1,2,3,4-tetrahydro-, kinetics of thermolysis / Bicyclo[3.2.0]hept-6-ene derivatives / Benzocycloheptene derivatives

On the Thermal Rearrangement of the Bicyclo[1.1.0]butane System. A Kinetic Investigation of the Conversion of Tricyclo[4.1.0.0 $\left.0^{2.7}\right]$ heptanes into Bicyclo[3.2.0]hept-6-enes

Tricyclo[4.1.0.0.7 $]$ heptane (7), its 1- (20) and 2-phenyl derivatives (22), tetracyclo[5.1.0.0.2, $.0^{3,5}$ ]octane (17), 1,2,3,4-tetrahydro1,2,3-methenonaphthalene (25) as well as its 1- (35), 2- (32), and 3-phenyl derivatives (27) have been thermolyzed in solution in the temperature range between 110 and $230^{\circ} \mathrm{C}$. The activation parameters of these reactions have been determined. The parent hydrocarbon 7 is converted into bicyclo[3.2.0]hept6 -ene (11) in high yield, and the other substrates behave analogously, i.e. the cyclobutene derivatives $18,21,23,26,28,33$, 34 , and 36 are formed. Arising from 27, the cyclobutene 28 is observed as intermediate, which is transformed rapidly to a mixture of the benzocycloheptenes 29 and 31 . To undergo this ring enlargement, the other cyclobutenes require more severe conditions. The thermal rearrangements of 20,22 , and 27 are accompanied by parallel reactions, which have been identified as acid-catalized processes in the case of 22 and 27 giving rise to the norcarene derivatives 24 and 30, respectively. In 2-phenylmethenonanpthalene 32 two pathways to a cyclobutene derivative exist; they are found to be followed in a $82: 18$ ratio. The relative reaction rates of all substrates investigated can be interpreted in terms of a consistent mechanistic model. Accordingly, the tricyclo[4.1.0.0 $\left.0^{2.7}\right]$ heptane system is transformed in a concerted but highly asynchronous process to an $(E, Z)-1,3-$ cycloheptadiene, which then undergoes a rapid conrotatory ring closure to yield the corresponding bicyclo[3.2.0]hept-6ene.

\section{A. Stand der Kenntnis}

Die Woodward-Hoffmann-Regeln ${ }^{[4 a]}$ und das Dewar-EvansPrinzip ${ }^{|4|}$ beschreiben die Thermolyse von Bicyclo[1.1.0]butan (1) ${ }^{[s]}$ zu 1,3-Butadien (2) als konzertierten Prozeß $\left(\left[{ }_{\sigma} 2_{a}+{ }_{\sigma} 2\right]\right.$-Reaktion), in dem sich die Offnung der einen Bindung disrotatorisch und die der anderen konrotatorisch vollzieht. Experimentelle Ergebnisse, die dieses Bild stützen, wurden durch kinetische Messungen zur Umlagerung von 1 und die Ermittlung der Stereochemie der Thermolyse der beiden Dimethylderivate $\mathbf{3 a}$, b erzielt.

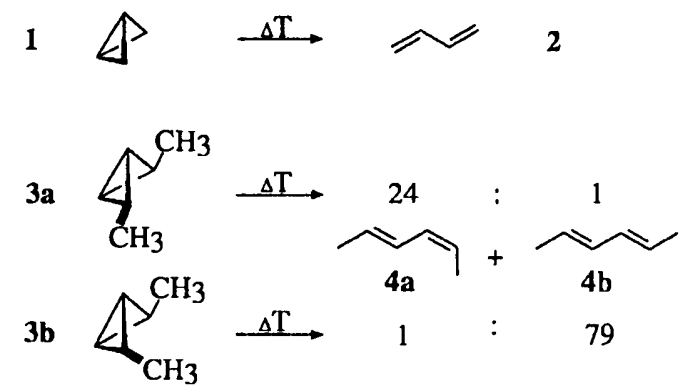

Frey und Stevens ${ }^{|6|}$ und Srinivasan et al. ${ }^{[7]}$ bestimmten die Aktivierungsenergie für den Übergang $1 \rightarrow 2$ zu $40.6 \mathrm{bzw} .41 .3 \mathrm{kcal}$ $\mathrm{mol}^{-1}$. Aufgrund thermochemischer Abschätzungen wurde das Diradikal 5 als Zwischenstufe des Wegs von 1 nach 2 ausgeschlossen ${ }^{[8.9]}$. Demnach sollte die Reaktionsenthalpie für $1 \rightarrow 5$
$49 \mathrm{kcal} \mathrm{mol}^{-1}$ betragen. Mit der gemessenen Aktivierungsenergie von ca. $41 \mathrm{kcal} \mathrm{mol}^{-1}{ }^{[6,7]}$ wäre also der Zustand 5 nicht erreichbar.

Die Thermolyse von exo,exo- (3a) und endo,exo-2,4-Dimethylbicyclobutan (3b) studierten Closs und Pfeffer ${ }^{[10]}$. Mit hohen Stereoselektivitäten bilden sich $(E, Z)$ - $(4 a)$ bzw. $(E, E)-2,4-$ Hexadien (4b), wie durch die Theorie der konzertierten Reaktionen ${ }^{[4]}$ gefordert. Die Anteile am jeweils unerwarteten 2,4-Hexadien (4a:4b $=$ 24:1 bzw. 1:79) mögen ebenso wie diejenigen an anderen Produkten ${ }^{[10]}$ gering erscheinen, jedoch nahmen Dewar und Kirschner ${ }^{[11]}$ die stereochemische Leckage als experimentellen Beleg für das Auftreten von zu 5 verwandten Diradikaloiden, die aufgrund semiempirischer Rechnungen Zwischenstufen seien. In diesen Spezies bestehe eine Wechselwirkung zwischen den beiden Radikalzentren, die pyramidalisiert seien, weshalb die Information der Konfiguration der Ausgangsverbindung erhalten bleibe. Daher erfolge die Weiterreaktion - Offnung des zweiten Dreirings - überwiegend gemäß der Theorie der konzertierten Prozesse ${ }^{(4)}$. Der Diradikalcharakter dieser Teilchen sei aber ausreichend für die Isomerisierung des Zentrums in der Seitenkette, was zum „verbotenen" Diradikaloid führen könne mit der Konsequenz, daß die Ringöf nung nun anders als vorher verlaufe, d.h. das ,verbotene“ Produkt hervorbringe. Zahlenmäßig betrage der Unterschied der Aktivierungsenergien zwischen „erlaubtem“ und „verbotenem“ Weg nur ca. $2 \mathrm{kcal} \mathrm{mol}^{-1}{ }^{[1]}$, ein Wert, der mit den Ergebnissen von Closs und Pfeffer ${ }^{[10]}$ bestens im Einklang steht. Die gleichen semiempirischen Rechnungen liefern mit dem 5 nahestehenden Diradikaloid als $\mathrm{Z}$ wischenstufe auch eine nahe am experimentellen Wert ${ }^{[6,7]}$ liegende Aktivierungsenergie des Übergangs $1 \rightarrow 2^{[11]}$. 
<smiles>CC1CC1</smiles>

6

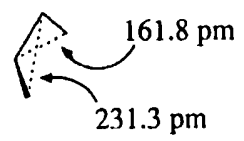

Aufgrund ihrer Rechnungen betrachten Shevlin und McKee ${ }^{[9]}$ die Umlagerung $1 \rightarrow 2$ zwar als konzertiert ( $\left[\sigma_{\mathrm{a}}+\sigma_{\mathrm{a}} \mathrm{2}\right]$-Reaktion), aber in hohem Maße asynchron. Im Übergangszustand (6), dessen Enthalpie $43.6 \mathrm{kcal} \mathrm{mol}^{-1}$ über der von 1 liege, sei eine der zu spaltenden Bindungen schon stark gestreckt $(231.3 \mathrm{pm})$, aber die Länge der anderen kaum vergrößert (161.8 pm). Zwischen $6^{(9)}$ und Dewars Diradikaloid ${ }^{[11]}$ scheint kein großer Unterschied zu bestehen. Jedoch betonen die Autoren ${ }^{[9]}$, daß das Diradikal 5 eine um $5.7 \mathrm{kcal} \mathrm{mol}^{-1}$ höhere Energie als 6 habe. Im Falle geeigneter Substituenten könnte aber ein zu 5 analoges Diradikal erreichbar sein.

Somit kann man wenigstens drei mechanistische Alternativen unterscheiden, nämlich erstens den konzertierten synchronen, zweitens den konzertierten, aber stark asynchronen und drittens den zweistufigen Prozeß mit einem intermediären Diradikal.

Wir wollten nun Substituenteneffektc auf die Umlagerungsgeschwindigkeit studieren. Jedoch sind die Zugänge zum Grundkörper 1 schon etwas mühevol[ ${ }^{[12]}$, womit die Bereitung geeignet substituierter Derivate zumindest als schwierig erschien. Wir wandten uns daher dem Tricyclo[4.1.0.0.7. $]$ heptan (7) zu, von dem zahlreiche Abkömmlinge mit dem von Moore et al. ${ }^{[13]}$ durch die Synthese von 7 eingeführten Verfahren aus den Dibromcarben-Addukten von $\mathrm{Cy}$ clohexenen dargestellt worden sind ${ }^{[14]}$.

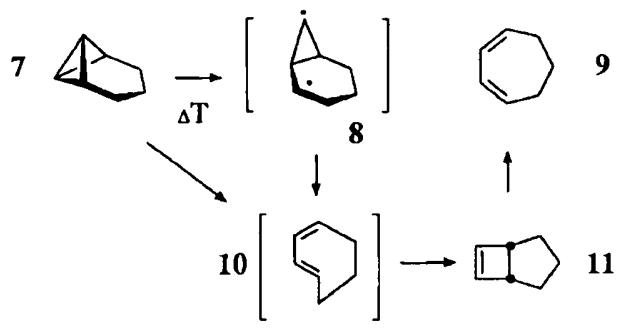

Wiberg und Szeimies ${ }^{[15]}$ thermolysierten 7 in der Gasphase und erhielten Gemische aus Bicyclo[3.2.0]hept-6-en (11) und $(Z, Z)-1,3$ Cycloheptadien (9). Mit sinkender Temperatur wurde der Anteil an 9 kleiner (größtes Verhältnis 11:9 = 91:9), so daß 9 wohl als Folgeprodukt von 11 zu betrachten ist. Als Aktivierungsenergie für den Übergang $11 \rightarrow 9$ wurden $45.9 \mathrm{kcal} \mathrm{mol}^{-1} \mathrm{ermittelt}^{[16]}$. Der einstufige Ablauf $7 \rightarrow 11$ ist ,verboten" ${ }^{[4]}$, weshalb die Autoren $(E, Z)-1,3$ Cycloheptadien (10) als Zwischenstufe postulierten, die im zweiten, rascheren Schritt dem konrotatorischen Ringschluß zu 11 unterliege.

Obwohl die direkte Bildung von 10 aus 7 wie die Umlagerung $3 \mathbf{a} \rightarrow 4 \mathbf{a}$,erlaubt" ist ${ }^{(4)}$, wurde auch der zweistufige Weg über das Diradikal 8 in Erwägung gezogen, aber aufgrund semiempirischer Rechnungen verworfen ${ }^{[15]}$. Beide Arten der Offnung des Dreirings von $8 \mathrm{zu}$ einem Dien, d.h. disrotatorisch zu 9 und konrotatorisch zu 10, seien ,erlaubt" und unterschieden sich kaum in der Aktivierungsenergie. Daher erkläre der konzertierte Schritt $7 \rightarrow \mathbf{1 0}$ das Ergebnis der selektiven Bildung von 11 am besten ${ }^{[15]}$. Die beiden Ringöffnungsmoden von $\mathbf{8}$, in dem die Wechselwirkung der beiden Radikalzentren berücksichtigt wurde und das nach Dewar als Diradikaloid zu bezeichnen wäre, untersuchten Dewar und Kirschner ${ }^{111}$, wie schon erwähnt, am Beispiel der Umlagerung von 3a,b noch einmal.

Wir haben dic Kinetik der Umlagerung von 7 in 11 in Lösung gemessen und dabei eine im Vergleich zur Thermolyse von 1 etwas klcinere Aktivierungsenergie (38.8 $\left.\mathrm{kcal} \mathrm{mol}^{-1}\right)$ gcfunden ${ }^{[17 \mathrm{a})}$. Aller- dings störte dabei die trotz des Lösungsmittels $N, N, N^{\prime}, N^{\prime}$-Tetramethylethylendiamin (TMEDA) durch eine Säurekatalyse ausgelöste Bildung von 2-Norcaren ${ }^{[13]}$. Eine kleine Menge 9 im Produktgemisch wäre unentdeckt geblieben. Homobenzvalen (12) verhält sich wie 7 und geht beim Erhitzen in Bicyclo[3.2.0]hepta2,6-dien (14) ohne Verunreinigung an 1,3,5-Cycloheptatrien über. Markierungsexperimente zeigten, daß als Zwischenstufe $(E, Z, Z)$ (13) und nicht $(Z, E, Z)-1,3,5-$ Cycloheptatrien anzunehmen ist ${ }^{[17 a !}$. Homobenzvalenon (Tropovalen) ${ }^{(17 b)}$ und sein 4-Acetoxyderivat ${ }^{(17) !}$ lagern ebenfalls in Bicyclo[3.2.0]hepta-2,6-dien-Derivate um, jedoch sind dabei $(Z . E, Z)$-Tropone als Intermediate zu vermuten.

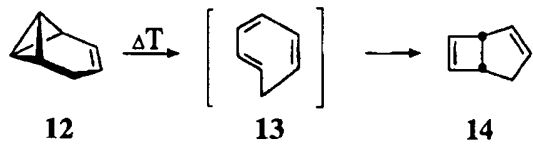

Die Vinylen-Einheit in 12 fördert die Umlagerung des Bicyclobutan-Teils erheblich, wie aus der im Vergleich zu 7 um $6.4 \mathrm{kcal}$ $\mathrm{mol}^{-1}$ niedrigeren Aktivierungsenergie folgt ${ }^{[17 a]}$ (neuer Wert: 5.2 $\mathrm{kcal} \mathrm{mol}^{-1}$, siehe Tab. 1). Unter dem Eindruck der Arbeit von Dewar und Kirschner ${ }^{[11]}$ führten wir diese Beschleunigung darauf zurück, daß das zu 8 analoge Diradikal auf dem Weg zu 13 Zwischenstufe sei. Die Allylmesomerie dieses Intermediats erkläre dessen Stabilisierung und damit auch die des geschwindigkeitsbestimmenden Übergangszustands, wenngleich der Betrag der Stabilisierung eigentlich als zu gering erschien, da dieser für das Allylradikal mit $12-13 \mathrm{kcal} \mathrm{mol}^{-1}$ angegeben wird ${ }^{[18]}$. Tropovalen reagiert langsamer als 12 (Faktor 85 bei $150^{\circ} \mathrm{C}, E_{\mathrm{a}}=31.2 \mathrm{kcal} \mathrm{mol}^{-1}$, $\lg A=(11.0)^{[17 b]}$.

Hasselmann und Loosen ${ }^{[19]}$ crmittelten die Aktivierungsparameter der thermischen Umlagerung von 3-Methylentricyclo[4.1.0.0.2.7heptan (15), die in Lösung weit überwiegend zum zugehörigen Cyclobuten und in geringem Ausmaß zu dessen Folgeprodukten 5- und 6-Methylen-1,3-cycloheptadien führt. Wie bei 12 so ist auch bei 15 die Reaktion wegen der Konjugation des Bicyclobutan-Systems mit der Ethylen-Einheit gegenüber 7 erheblich beschleunigt (siehe Tab. 1).
15

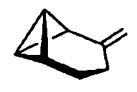

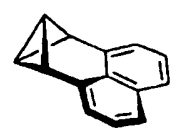

16
Pagni et al. ${ }^{[20]}$ stellten am Übergang des Methenophenalens 16 in das entsprechende Cyclobuten fest, da $B$ auch die Konjugation des Bicyclobutan-Systems mit einer Naphthalin-Einheit die Thermolyse im Vergleich zu 7 erheblich erleichtert (siehe Tab. 1).

Auf der Basis des Syntheseprinzips für $7^{[13]}$ haben wir nun Phenylderivate von 7 und 1,2,3,4-Tetrahydro-1,2,3-methenonaphthalin (25) maßgeschneidert, was in der vorangehenden und einer früheren Arbeit beschrieben ist ${ }^{[21]}$, um durch Messung der Umlagerungsgeschwindigkeiten eine Antwort auf die Frage zu bekommen, welcher der drei oben genannten Mechanismen die Wirklichkeit am besten beschreibt. Phenylderivate wurden aus zwei Gründen gewählt: erstens waren keine synthetischen Probleme zu befürchten ${ }^{[2]}$, und zweitens wirken Phenylgruppen nicht nur an Radikalzentren aufgrund der Benzylmesomerie stabilisierend ${ }^{[18\}}$, was in gleicher Weise auf diradikalische $Z$ wischenstufen zutrifft ${ }^{[22]}$, sondern können auch konzertierte Reaktionen beschleunigen, wenn sie an Zentren gebunden sind, die an Bindungsbruch und -neubildung beteiligt sind, so z. B. Phenylgruppen an C-3, -4 von Cyclobuten bei der thermischen Isomerisierung zu 1,3-Butadien ${ }^{[23]}$ (vgl. Tab. 4). 
Tab. 1. Geschwindigkeitskonstanten und Aktivierungsparameter für die Umlagerung von Tricyclo[4.1.0.0 $\left.0^{2.7}\right]$ heptanen in Bicyclo[3.2.0]hept-6-ene; die Fehlerangaben sind Standardabweichungen

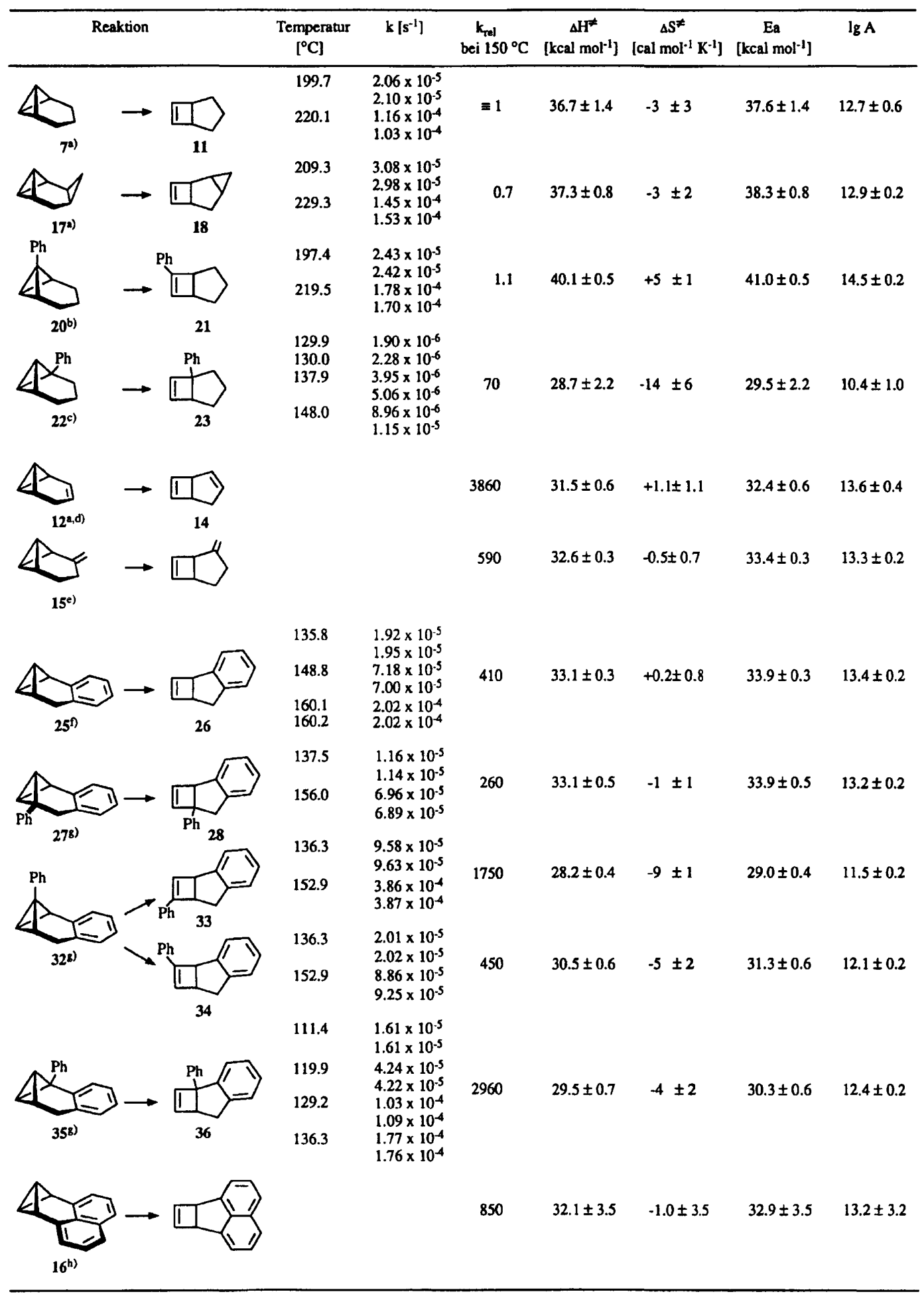

a) Losungsmittel: $\left[D_{8}\right]$ Toluol mit 5-10\% N,N,N, $N^{\prime}$-Tetramethylethylendiamin (TMEDA) und Mesitylen als internem Standard für die quantitative Analyse. - b) Lösungsmittel: $\left[D_{6}\right]$ Benzol mit 20\% Cyclohexan als Locksubstanz für das NMR-Spektrometer und Cyclohexen als internem Standard. - c) Losungsmittel: $\left[D_{6}\right]$ Benzol mit 5\% TMEDA, 20\% Cyclohexan und Anisol als internem Standard. - d) Lit. ${ }^{174)}$. - c) Lit. 19), Losungsmittel: $n$-Heptan. . f) Lösungsmittel: $\left[D_{6}\right]$ Benzol mit 5\% TMEDA und Mesitylen oder Cyclohexan als internem Standard. - 8) Losungsmittel: Gemische aus 5-70\% [D $]$ Benzol und 95-30\% TMEDA sowie Cyclohexen als internem Standard. - ") Lit. ${ }^{20)}$, Losungsmittel: Cyclohexan. 


\section{B. Ergebnisse}

B.1. Thermolyse von Tricyclo[4.1.0.0 $\left.0^{2,7}\right]$ heptan (7) und seiner Derivate 17, 20 und 22

Unter veränderten Bedingungen wurde die Messung der Kinetik der Umlagerung des Tricycloheptans 7 wiederholt. Nach Lagerung einer Mischung aus 7, Mesitylen als internem Standard, TMEDA und $\left[\mathrm{D}_{8}\right]$ Toluol als Lösungsmittel über $\mathrm{LiAlH}_{4}$ und durch die Gasphase erfolgenden Transfer in das NMR-Röhrchen, das im Vakuum abgeschmolzen wurde, verlief die Thermolyse ohne störende Bildung von 2Norcaren. Es entstanden lediglich das Bicyclohepten 11 und 1,3-Cycloheptadien (9). Nach drei Halbwertszeiten war die Ausbeute praktisch quantitativ und das Verhältnis 9:11 lag bei 1.2:98.8. Letzteres wuchs bei fortgesetztem Erhitzen langsam aber stetig an. Mit noch höherer Wahrscheinlichkeit als früher ${ }^{[15]}$ charakterisieren diese Befunde 9 als Folgeprodukt von 11. Geschwindigkeitskonstanten und Aktivierungsparameter für den Übergang $7 \rightarrow 11$ zeigt Tab. 1 .

Bei gleicher Probenvorbereitung, aber in Abwesenheit von TMEDA lagerte 7 bei $200^{\circ} \mathrm{C}$ in ein $1.4: 1.0$-Gemisch aus 11 und 3-Methylen-1-cyclohexen um. Das letztere Produkt entsteht mit hoher Selektivität bei der Einwirkung des Dicarbonylrhodiumchlorid-Dimers auf $7^{[24]}$. Analog wird aus 15 beim Erwärmen in Benzol in Gegenwart von Kupfer zu $81 \%$ 3,6-Dimethylen-1-cyclohexen gebildet ${ }^{[19]}$. Somit wirkt TMEDA bei der Thermolyse von 7 wohl als Komplexbildner für Spuren von Schwermetall-Ionen.

Uber die thermische Umwandlung von Tetracyclo[5.1.0.0.4.4. $0^{3.5}$ ] octan (17) unter Bedingungen, wie für 7 oben angegeben, hatten wir kurz berichtet ${ }^{[25 a]}$. Sie führt abweichend vom Ergebnis der Gasphasenpyrolyse ${ }^{[26]}$ hauptsächlich zu trans-Tricyclo[4.2.0.0 $0^{2,4}$ ]oct-7-en (18), das durch sein charakteristisches ${ }^{1} \mathrm{H}-\mathrm{NMR}-S$ pektrum ${ }^{[27]}$ identifiziert wurde.

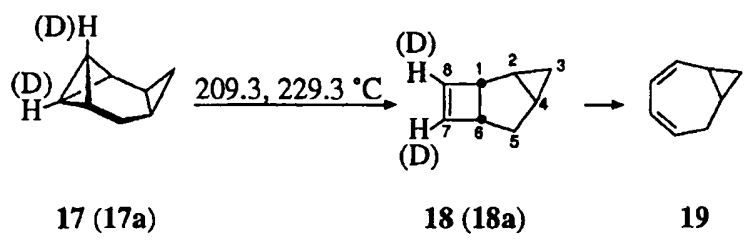

Die kinetischen Messungen zur Umlagerung von 17 wurden bei 209.3 und $229.3^{\circ} \mathrm{C}$ ausgeführt. Geschwindigkeitskonstanten und Aktivierungsparameter enthält Tab. 1. Nach einer Halbwertszeit lag die Ausbeute an 18, bezogen auf umgesetztes 17 , bei $80-95 \%$, nach zwei Halbwertszeiten bei $73-81 \%$. Die neben denen von $18 \mathrm{im}{ }^{1} \mathrm{H}-\mathrm{NMR}-$ Spektrum vorhandenen Banden passen zwanglos zu 2,3-Homotropiliden (19) ${ }^{[28]}$. Somit vollzieht sich die Ringerweiterung $18 \rightarrow 19$ mit ähnlicher Geschwindigkeit wie der Übergang $11 \rightarrow 9$. Bei der Ermittlung des Endwerts der kinetischen Messungen durch längeres Erhitzen auf $230^{\circ} \mathrm{C}$ veränderte sich das NMR-Spektrum der Probe weiter. Die Signale von 18 verschwanden allmählich vollständig, und es trat mit geringer Intensität das scharfe Singulett von Benzol auf, das als Produkt der Thermolyse von 19 bekannt ist ${ }^{[29]}$. Als charakteristische Hauptbanden entwickelten sich ein Quartett bei $\delta=-0.58$, bezogen auf internes Benzol, mit $J=$
$4.3 \mathrm{~Hz}$, dessen Lage für das Kohlenstoffgerüst von 18 als Strukturelement spricht, und ein Pseudotriplett bei $\delta=$ 6.44, das typisch für ein 3,6-überbrücktes Cyclohexen-Derivat und dem entsprechenden Signal des [4 + 2]-Cycloadditionsdimers von 1,3-Cyclohexadien sehr ähnlich ist. Vermutlich handelt es sich um das Diels-Alder-Addukt von 18 an das Diensystem von 19. Ein Produkt dieses Typs fanden wir auch bei der Thermolyse von hochkonzentriertem Benzvalenoxid; es entsteht wohl durch Addition des primären Umlagerungsprodukts (2-Oxabicyclo[3.2.0]hepta-3,6-dien) an ein sekundäres (Benzoloxid) ${ }^{[30]}$. Erhitzen des $\left[D_{2}\right]-$ Tetracyclooctans 17a führte selektiv zur Bildung des $\left[\mathrm{D}_{2}\right]$ Tricyclooctens $\mathbf{1 8 a}$.

1-Phenyltricyclo[4.1.0.0.7.7 $0^{2.0 p t a n ~(20) ~ w a r ~ v o n ~ F u j i t a ~ e t ~}$ al. ${ }^{[31]}$ in der Gasphase bei $450^{\circ} \mathrm{C}$ pyrolysiert worden. Sie hatten 2-Phenyl-1,3-cycloheptadien als einziges Produkt erhalten und dieses durch Belichten in 6-Phenylbicyclo[3.2.0]hept-6-en (21) umgewandelt.
20

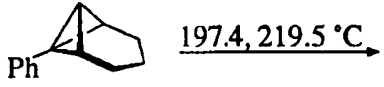

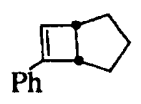

21
Wir führten jetzt die Thermolyse von $20 \mathrm{im}$ Gemisch aus [D $]$ Benzol, Cyclohexan und Cyclohexen bei 197.4 und $219.5^{\circ} \mathrm{C}$ durch und kamen nicht zu 2-Phenyl-1,3-cycloheptadien, sondern zu 21 als Hauptprodukt. Bei Umsätzen von 80-91\% lagen die Ausbeuten an 21, bezogen auf umgesetztes 20 , bei $48-82 \%$. Daneben entstand in unterschiedlichen Mengen eine zweite Verbindung, die nicht identifiziert werden konnte. Ihre Bildung ließ sich durch Zusatz von TMEDA nicht unterdrücken. Umsatzdiagramme belegen, daß das zweite Produkt nicht aus 21 in einer Folgereaktion hervorgeht. Daher wurde die Abnahme der Konzentration von 20 im Sinne von Parallelreaktionen 1. Ordnung behandelt. Die so erhaltenen Geschwindigkeitskonstanten und Aktivierungsparameter für die Umlagerung $20 \rightarrow 21$ enthält Tab. 1.

2-Phenyltricyclo[4.1.0.0 $\left.0^{2.7}\right]$ heptan (22) hatten wir erstmals dargestellt ${ }^{[21 a]}$. Da die Abtrennung von 22 von den Nebenprodukten 3-Phenyltricyclo[4.1.0.0 $\left.0^{2,7}\right]$ heptan, 6-Phenyltricyclo[3.2.0.0 2.7 $]$ heptan und 1-Phenylbicyclo[3.2.0]hept-6-en (23) nicht gelang, thermolysierten wir das Gemisch nach sorgfältiger Probenvorbereitung in einer Mischung aus $\left[D_{6}\right]$ Benzol, Cyclohexan, Anisol und TMEDA. Bei $129.9-148.0^{\circ} \mathrm{C}$ veränderten sich die Nebenprodukte nicht, und 22 ging sowohl in 23 als auch 2-Phenyl-2-norcaren (24) über. Das Verhältnis 23:24 war während eines Versuchs und auch bei verschiedenen Versuchen nicht konstant. Nach kurzer Reaktionszeit lag es bei 1.17-0.66 und am Ende der Versuche bei $0.90-0.47$, d.h. überwiegend bildete sich 24 mit 23:24 = 32:68 im Extremfall.

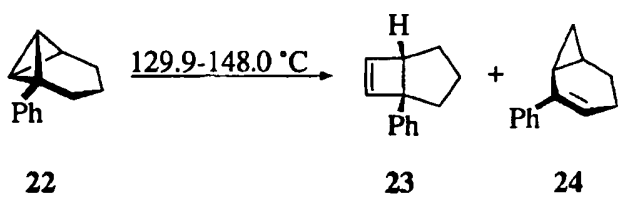


Die Entstehung von 24 ist das Ergebnis einer Säurekatalyse ${ }^{[21 a]}$, obwohl das Reaktionsgemisch so gut wie möglich getrocknet wurde und TMEDA enthielt. Ursache für das leichte Ablaufen dieses wohl schon durch die Glasoberfläche katalysierten Prozesses ist die große Stabilität des aus 22 durch Anlagerung eines Protons an ein BicyclobutanBrückenkopf-C-Atom hervorgehenden 2-Phenyl-2-norcaranyl-Kations, das sich durch Abgabe eines Protons von C3 in 24 umwandelt. Die Isomerisierung $22 \rightarrow 24$ ist analog zur Säure-induzierten Umlagerung von 7 in 2-Norcaren ${ }^{[13]}$.

Anders als 24 ist 23 das erwartete Produkt der thermischen Umlagerung von 22. Unter der Annahme, daß aus 22 nur 23 und 24 entstanden, lag die Ausbeute an 23 bei $32-47 \%$. Fujita et al. ${ }^{[31]}$ hatten 23 erstmals beschrieben, und zwar als Produkt der Belichtung von sowohl 20 als auch 1-Phenyl-1,3-cycloheptadien.

Das hohe Ausmaß der Bildung von 24 und die Zunahme seines Anteils beim Fortgang der Thermolyse beeinträchtigten die Ermittlung der Geschwindigkeitskonstanten für die Umlagerung $22 \rightarrow 23$ erheblich. Angesichts der Varianz des 23: 24-Verhältnisses ist die Behandlung der Prozesse als $\mathrm{Pa}$ rallelreaktionen 1 . Ordnung nur eine sehr grobe Näherung. Die Geschwindigkeitskonstanten in Tab. 1 sind daher mit größeren Fehlern behaftet als in den anderen Fällen und die Aktivierungsparameter weniger zuverlässig als die Standardabweichung angibt, weil bei deren Berechnung die Fehler der Geschwindigkeitskonstanten nicht eingingen.

B.2. Thermolyse von 1,2,3,4-Tetrahydro-1,2,3methenonaphthalin (25) und seiner Phenylderivate 27, 32 und 35

Weil die thermischen Umlagerungen der Phenylderivate 20 und 22 von 7 von Parallelreaktionen begleitet sind und die Herstellung von 7-Abkömmlingen mit zwei Phenylgruppen am Bicyclobutan-System außer einer Probe noch stark verunreinigten 2,6-Diphenyltricyclo[4.1.0.0 $\left.0^{2.7}\right]$ heptans nicht gelang $^{\text {[21a] }}$, wandten wir uns dem 1,2,3,4-Tetrahydro-1,2,3methenonaphthalin-System zu. Die Stammsubstanz 25 war bekannt ${ }^{[32]}$, und die drei Derivate mit je einer Phenylgruppe in den drei verschiedenen Positionen seines BicyclobutanSystems $(27,32,35)$ konnten wir aufbauen ${ }^{[21]}$.

$$
252_{3}^{26}
$$

Murata et al. ${ }^{[32]}$ hatten aus 25 in Tetrachlormethan bei $190-200^{\circ} \mathrm{C}$ ein $72: 28-G e m i s c h$ aus 3,7b-Dihydro-2a $H$-cyclobut $[a]$ inden (26) und $5 H$-Benzocyclohepten erhalten. Wir thermolysierten jetzt 25 in einem Gemisch aus [ $\left.D_{6}\right]$ Benzol, TMEDA und Mesitylen oder Cyclohexan, wobei mit quantitativer Ausbeute 26 entstand. Die Geschwindigkeit der Umlagerung wurde bei $135.8-160.2^{\circ} \mathrm{C}$ bis zu Umsätzen von $82-91 \%$ gemessen. Die Auswertung als Reaktion 1. Ordnung erbrachte die geforderte Abhängigkeit der Konzentration von der Zeit. In Tab. 1 sind die Geschwindigkeitskonstanten und die Aktivierungsparameter zusammengestellt.
Ein weniger eindeutiges Ergebnis hatte die Thermolyse von 1,2,3,4-Tetrahydro-3-phenyl-1,2,3-methenonaphthalin (27) bei 137.5 und $156.0^{\circ} \mathrm{C}$, denn es entstanden die Benzocycloheptene 29 und 31 sowie das Dihydrocyclopropanaphthalin 30 mit Ausbeuten von $59-70 \%, 7-22 \%$ und $11-23 \%$. Bei $156^{\circ} \mathrm{C}$ erwies sich der Anteil von 30 als abhängig von der TMEDA-Konzentration in der Lösung. Bei ca. 50\% TMEDA war das Minimum von 30 erreicht. Diese Abhängigkeit kennzeichnet die Bildung von $\mathbf{3 0}$ als Säurekatalysierten Prozeß. Dementsprechend erhielten wir $\mathbf{3 0}$ und daneben ein wenig 29 auch bei der Behandlung von $27 \mathrm{mit}$ Trifluoressigsäure oder Tetracyanethylen. Das letztere Reagenz benutzten wir mit der Absicht, ein Addukt aus 27 herzustellen in Analogie zu den Reaktionen von 7 und 12 mit Tetracyanethylen ${ }^{[33]}$. Als Zwischenstufe auf dem Weg zu 30 ist wie bei der Bildung von 24 aus 22 ein Derivat des 2Norcaranyl-Kations mit einer Phenylgruppe am kationischen Zentrum anzunehmen. Die NMR-Spektren belegen die Struktur von 30. Insbesondere sprechen die ${ }^{1} \mathrm{H}-\mathrm{NMR}-$ Daten klar für eine cis-vicinal-substituierte CyclopropanEinheit.

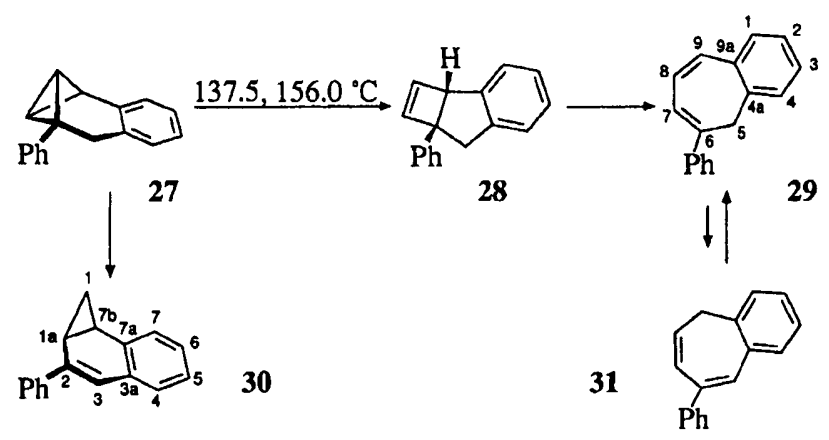

Das Verhältnis 29:31 war während der Reaktion nicht konstant. Am Anfang konnten wir nur 29 beobachten, während am Ende wohl ein Zustand nahe dem Gleichgewicht der Isomeren vorlag. Damit ist zu vermuten, daß 31 aus 29 durch $[1,5]-\mathrm{H}-$ Wanderung hervorging. Eine Umlagerung dieser Art läuft bei 1,3,5-Cycloheptatrien im gleichen Temperaturbereich $\mathrm{ab}^{[34]}$.

Der Ein-Stufen-ProzeB $27 \rightarrow 29$ ist wie $7 \rightarrow 9$ verboten ${ }^{[4]}$. Tatsächlich war ein Zwischenprodukt nachweisbar, dem wir aufgrund der charakteristischen chemischen Verschiebungen und Kopplungskonstanten im ${ }^{1} \mathrm{H}$-NMR-Spektrum (Tab. 2) die Struktur des Dihydrocyclobutindens $28 \mathrm{zu}-$ schreiben. Die im Vergleich zu 26 leicht eintretende Ringerweiterung $28 \rightarrow 29$ war zu erwarten, da die Phenylgruppe von 28 das für diesen Prozeß als Zwischenstufe anzunehmende Diradikal ${ }^{[35]}$ zusätzlich stabilisiert und damit auch die Aktivierungsbarriere absenkt.

Auch das Verhältnis $(28+29+31): 30$ blieb im Verlauf eines Versuchs nicht konstant, so daß ähnliche Gegebenheiten wie bei der Thermolyse von 22 hinzunehmen waren. Allerdings war die Bildung von $\mathbf{3 0}$ aus 27 eindeutig Nebenreaktion und spielte daher eine weniger bedeutende Rolle als die Umlagerung $22 \rightarrow$ 24. Die Ermittlung der Geschwindigkeitskonstanten des Prozesses $27 \rightarrow 28$ durch Behand- 
Tab. 2. ${ }^{1} H$-NMR-Chemische Verschiebungen ( $\delta$-Werte) und Kopplungskonstanten (in Hz) sowie Signalmultiplitzităten von Bicyclo[3.2.0]hept-6en-Derivaten in $\mathrm{CDCl}_{3}$, wenn nicht ein anderes Solvens angegeben ist. Die 6-, 7-H-Signale der Bicyclen und die 1-, 2-H-Signale der Tricyclen zeigen kleine Kopplungen $(\leq 1 \mathrm{~Hz})$ zu den Brückenkopf- $\mathrm{H}$ und/oder $\mathrm{CH}_{2}$ Gruppen. Die Anzahl dieser Kopplungen geht zum Teil aus den Multiplitzitätsangaben hervor
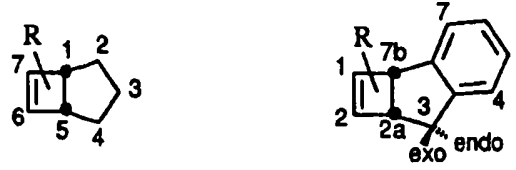

a) Lösungsmittel: $\left[D_{8}\right]$ Toluol/TMEDA mit Mesitylen als internem Standard (Signal bei $\left.\delta=6.80\right)$. - b) Nicht ermittelt. - c) Linienabstand des "d" $\approx 6 \mathrm{~Hz}$. - d) In Lit. ${ }^{31)}$ finden sich in $\mathrm{CCl}_{4}$ ermittelte Daten. - e) Zuordnung austauschbar. - ${ }^{n}$ Einige der angegebenen Kopplungskonstanten weichen von denen einer früheren Analyse ${ }^{42 \mathrm{~b}}$ ) erheblich ab. ${ }^{-8}$ ) Entkopplungsversuche belegen $J_{3 e n d o, 7 b}=1.5, J_{3 e x o, 7 b}=0.5 \mathrm{~Hz}$. h) $\mathrm{Lob}-$ sungsmittel: TMEDA, $\left[D_{6}\right]$ Benzol mit Cyclohexen als internem Standard (Signal bei $\delta=5.70$ ). - i) Zuordnung austauschbar. - j) Von den Signalen von 29 - 31 überlagert.

lung der Abnahme der Konzentration von 27 als Parallelreaktionen 1 . Ordnung muß folglich als grobe Näherung betrachtet werden. Außerdem wurde angenommen, daß 29 und 31 ausschließlich via 28 entstehen, was Zeit-UmsatzDiagramme nahelegen. Sollte ein Teil von 29 und damit auch 31 wie 30 durch Säure-Katalyse aus 27 gebildet werden, wobei es sich aber nur um einen kleinen Teil von 29 handeln könnte, weil Trifluoressigsäure neben $\mathbf{3 0}$ nur wenig 29 aus 27 generierte, dann sind die Geschwindigkeitskonstanten der Tab. 1 obere Grenzwerte.<smiles>c1ccc(C2C3c4ccccc4C4CC3C42)cc1</smiles>

32

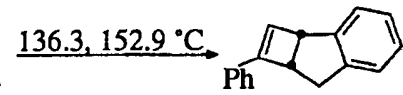

33

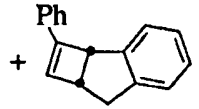

34
Mit besonderem Interesse thermolysierten wir 1,2,3,4-Tetrahydro-2-phenyl-1,2,3-methenonaphthalin (32), weil hierdurch die Frage nach der Chemoselektivität beantwortet werden konnte. Die Umlagerung führten wir bei 136.3 und $152.9^{\circ} \mathrm{C}$ aus und erhielten in der Tat zwei Produkte, nämlich die Phenylcyclobutindene 33 und 34 im Verhältnis 82:18. Nebenprodukte wurden nicht beobachtet, und die ${ }^{1} \mathrm{H}$ NMR-spektroskopisch bestimmten Ausbeuten lagen über 90\%. Das Verhältnis 33:34 war bei jedem der Versuche während der gesamten Meßzeit konstant, weshalb die $\mathrm{Ge}$ schwindigkeitskonstanten für die Bildung von 33 und $34 \mathrm{im}$ Sinne von Parallelreaktionen 1. Ordnung ermittelt wurden. Sie finden sich ebenso wie die Aktivierungsparameter in Tab. 1.

Die Zuordnung der Struktur 33 zum Hauptprodukt erfolgte anhand der NMR-Daten, und zwar im wesentlichen auf der Basis der ${ }^{13} \mathrm{C}$-NMR-chemischen Verschiebungen (Tab. 3), wobei die Phenylsubstituenten-Effekte in 21 und 23 relativ zu 11 eine wichtige Voraussetzung lieferten. So beträgt der $\alpha$-Effekt in 2313.6 und der $\beta$-Effekt auf C-5 $8.0 \mathrm{ppm}$. Wendet man diese Werte auf das Paar 26/36 an, so ergibt sich die in Tab. 3 angegebene Zuordnung von $C$ $2 \mathrm{a}$ und $\mathrm{C}-7 \mathrm{~b}$ von 26 . In 21 wurden nun dem $\beta$-Effekt auf $\mathrm{C}-5$ -2.1 und dem $\gamma$-Effekt auf $C-1-4.4$ ppm zugeschrieben. Dies ist aufgrund der Effekte der Phenylgruppe in 2- ${ }^{\text {[36a] }}$ und 1-Phenylpropen ${ }^{[36 \mathrm{~b}]}$ auf das Methyl-C-Atom relativ zu 
Tab. 3. ${ }^{13} \mathrm{C}$-NMR-Chemische Verschiebungen ( $\delta$-Werte) und in einigen Fällen ${ }^{13} \mathrm{C}-\mathrm{H}-\mathrm{Kopplungskonstanten} \mathrm{über} \mathrm{eine} \mathrm{Bindung} \mathrm{(in} \mathrm{Hz}$, jeweils zweite Zeile) von Bicyclo[3.2.0]hept-6-en-Derivaten in $\mathrm{CDCl}_{3}$ oder $\mathrm{C}_{6} \mathrm{D}_{6}(33,34)$. Die angegebenen Zuordnungen beruhen auf off-resonance-entkoppelten oder DEPT-Spektren sowie auf der Interpretation von Substituenteneffekten (siehe Text)
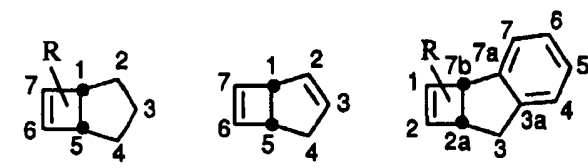

$11,21,23$
14
$26,33,34,36$

\begin{tabular}{|c|c|c|c|c|c|c|c|c|c|c|c|c|}
\hline Nr. & C-6 & C-7 & C-1 & C-5 & $\mathrm{C}-2$ & $\mathrm{C}-3$ & C-4 & & ipso-C & $0-\mathrm{C}$ & $m-\mathrm{C}$ & $p-\mathrm{C}$ \\
\hline 11 & $\begin{array}{l}137.5 \\
166\end{array}$ & $\begin{array}{c}137.5 \\
166\end{array}$ & $\begin{array}{l}48.1 \\
143\end{array}$ & $\begin{array}{r}48.1 \\
143\end{array}$ & $\begin{array}{l}26.7 \\
129\end{array}$ & $\begin{array}{l}23.2 \\
129\end{array}$ & $\begin{array}{l}26.7 \\
129\end{array}$ & & $\cdot$ & - & - & - \\
\hline 21 & 146.2 & $\begin{array}{c}128.0 \\
166\end{array}$ & $\begin{array}{l}43.7 \\
144\end{array}$ & $\begin{array}{l}46.0 \\
144\end{array}$ & $\begin{array}{l}26.0^{a)} \\
128\end{array}$ & $\begin{array}{l}23.4 \\
131\end{array}$ & $\begin{array}{l}26.5^{a)} \\
127\end{array}$ & & 134.1 & $\begin{array}{c}124.8 \\
158\end{array}$ & $\begin{array}{l}128.4 \\
157\end{array}$ & $\begin{array}{c}127.4 \\
161\end{array}$ \\
\hline 23 & 138.8 & 136.3 & 61.7 & 56.1 & 34.1 & 24.7 & 27.1 & & 146.1 & 126.2 & 128.1 & 125.7 \\
\hline \multirow[t]{2}{*}{14} & $\begin{array}{c}139.8 \\
167\end{array}$ & $\begin{array}{c}145.1 \\
168\end{array}$ & $\begin{array}{l}55.7 \\
143\end{array}$ & $\begin{array}{l}44.6 \\
143\end{array}$ & $\begin{array}{l}132.7^{a)} \\
163\end{array}$ & $\begin{array}{l}130.8^{2)} \\
160\end{array}$ & $\begin{array}{l}32.9 \\
128\end{array}$ & & - & - & - & - \\
\hline & $\mathrm{C}-2$ & C-1 & C-7b & $\mathbf{C}-2 \mathbf{a}$ & C-3a & C-7a & C-3 & $C-4,-5,-6,-7$ & & & & \\
\hline 26 & $\begin{array}{l}139.5 \\
170\end{array}$ & $\begin{array}{c}143.6 \\
171\end{array}$ & $\begin{array}{l}55.2 \\
144\end{array}$ & $\begin{array}{l}45.4 \\
144\end{array}$ & $144.0^{\mathrm{a})}$ & $144.1^{\text {a) }}$ & $\begin{array}{c}32.8 \\
130\end{array}$ & 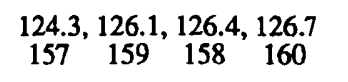 & - & - & - & - \\
\hline 33 & 149.0 & 133.77 & 51.3 & 44.0 & $143.9^{\text {a) }}$ & $144.8^{\text {a) }}$ & 32.2 & $124.8,126.6,126.7,127.0^{b)}$ & 133.84 & 125.3 & 128.6 & $128.3^{b}$ \\
\hline 34 & 129.9 & 153.5 & 53.9 & 41.4 & $143.6^{\text {a) }}$ & $145.0^{2)}$ & 33.7 & $125.6,126.4,126.8,127.2^{b)}$ & 134.1 & 125.2 & c) & $b, c)$ \\
\hline 36 & 138.0 & 144.5 & 67.9 & 55.5 & $143.6^{a)}$ & $143.8^{8)}$ & 32.6 & $124.9,126.2,126.3,126.5^{b)}$ & 147.0 & 126.9 & 128.1 & $127.1^{b}$ \\
\hline
\end{tabular}

a.b) Zuordnung austauschbar. - ${ }^{c)}$ Durch Banden von 33 und/oder das $C_{6} D_{6}$-Signal überlagert.

Propen ${ }^{[36 b]}$ gerechtfertigt: $\beta$-Effekt $=+2.4, \gamma$-Effekt $=-1.0$ ppm. Wenn auch in 21 beide Effekte abschirmend wirken, so ist die Zuordnung des größeren abschirmenden Werts zum $\gamma$-Effekt mit der üblichen Staffelung ${ }^{[36 \mathrm{~b}]}$ in Einklang. Auf dieser Grundlage ist die Wanderung der Signale von C2a und C-7b von 33 und 34 im Vergleich zu denen von 26 nach höherem Feld erwartet, ihr Abstand sollte aber bei 33 kleiner und bei $\mathbf{3 4}$ größer werden, was wir als Kriterium für die Zuordnung nutzten.

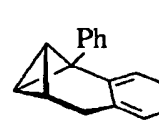

35

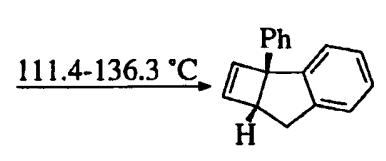

36

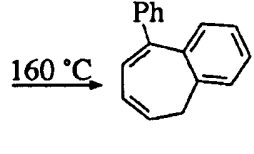

37
1,2,3,4-Tetrahydro-1-phenyl-1,2,3-methenonaphthalin (35) wurde bei $111.4-136.3^{\circ} \mathrm{C}$ umlagert. Als einziges Produkt bildete sich das Phenylcyclobutinden 36 mit Ausbeuten von über $88 \%$. Geschwindigkeitskonstanten und Aktivierungsparameter finden sich in Tab. 1. Längeres Erhitzen auf $160^{\circ} \mathrm{C}$ wandelte 36 mit 92\% Ausbeute in 9-Phenyl-5H-benzocyclohepten (37) um.

\section{Diskussion}

\section{C.1. Stereochemie}

Als Resultat aller Thermolysen ist festzuhalten, daß Tricyclo[4.1.0.0 2,7]heptan (7) und alle seine bisher untersuchten Derivate in Bicyclo[3.2.0]hept-6-en (11) bzw. Abkömmlinge davon übergehen. Wo andere Verbindungen auftreten, kann deren Bildung überzeugend entweder auf einen katalysierten Prozeß oder auf eine Folgereaktion der Thermolyseprodukte zurückgeführt werden. So gehen die neben 23 und 28 entstehenden 2-Norcaren-Derivate 24 bzw. 30 zweifellos durch Einwirkung von Säurespuren auf die Substrate 22 bzw. 27 hervor, die über die generelle Empfindlichkeit von Bicyclobutanen gegenüber Säuren ${ }^{[s]}$ hinaus offenbar besonders leicht durch solche isomerisiert werden, weshalb dieser Reaktionstyp trotz größter Sorgfalt bei der Probenvorbereitung nicht unterdrückt werden konnte. Andererseits müssen die Siebenringverbindungen 1,3-Cycloheptadien (9) und seine Derivate 19, 29 und 31 als Folgeprodukte der Bicyclo[3.2.0] hept-6-ene 11, 18 bzw. 28 betrachtet werden, die unter den Thermolysebedingungen erwartungsgemäß teilweise oder vollständig der Ringerweiterung unterliegen.

Somit erscheint die thermische Umlagerung von 7 und seinen Derivaten als stereospezifischer Prozeß. Akzeptiert man die Hypothese von Wiberg und Szeimies ${ }^{[15]}$, so sollten aus-

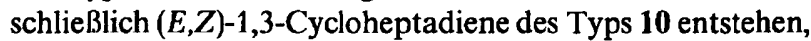
die dann zu cis-anellierten Cyclobutenen cyclisieren. Hält man die quantenmechanischen Rechnungen bezüglich der Öffnungsweise der Dreiringe in den Diradikaloiden $5^{[11]}$ und $\mathbf{8}^{[15]}$ für verläßlich, d.h. es bestehe bestenfalls ein kleiner Aktivierungsenergie-Unterschied zwischen beiden Moden, so schließt die eindeutige Stereochemie der hier untersuchten Reaktionen eine Diradikaloid-Zwischenstufe des Typs 8 aus. Dies folgt daraus, daß die von 5 abgeleiteten Diradikaloide, die Dewar und Kirschner ${ }^{[11]}$ bei der Umlagerung von 3a, b als Zwischenstufen annehmen, für „erlaubte" und „verbotene" Ringöffnung nur eine um ca. $2 \mathrm{kcal} \mathrm{mol}^{-1}$ differierende 
Barriere aufweisen sollen. Zweifellos wäre diese Differenz bei 8 verkleinert, weil der „verbotene“ Prozeß $(Z, Z)-1,3-C y-$ cloheptadien (9) lieferte, das viel stabiler ist als $(E, Z)-1,3-$ Cycloheptadien (10), das Produkt des ,erlaubten“ Wegs.

Die Stereospezifität der Reaktionen von 7 und seinen Derivaten steht nur in scheinbarem Widerspruch zu den $\mathrm{Er}$ gebnissen von Closs und Pfeffer ${ }^{[10]}$ mit 3a,b, denn dort könnten die geringen Mengen der 2,4-Hexadiene 4, die nicht auf ,erlaubtem" Weg entstehen, durchaus das Resultat einer Katalyse sein. Die Befunde, da $B$ aus beiden Ausgangsverbindungen in ähnlichem Ausmaß 1-Methyl-2-vinylcyclopropane gebildet werden, sprechen für eine Säurekatalyse. Die Behandlung von 27 mit Trifluoressigsäure erbrachte ein Ergebnis dieser Art, nämlich das Vinylcyclopropan 30 und das Dien 29, das Produkt der thermisch ,verbotenen“ Reaktion. Analog lassen sich die Produktgemische der Thermolysen von Hexamethyl-[37a] und 1,2,2,4,4-Pentamethylbicyclo[1.1.0]butan ${ }^{[37 b]}$ auf den gleichzeitigen Ablauf von thermischer und Säure-katalysierter Umlagerung zurückführen. Die für den einleitenden Schritt auf dem Weg zu den unerwarteten Verbindungen formulierten Diradikale ${ }^{[37 a, b]}$ halten wir für unwahrscheinlich.

\section{C.2. Geschwindigkeitskonstanten und Aktivierungsparameter}

Die in Tab. 1 zusammengestellten kinetischen Daten wurden in unterschiedlichen, jedoch in allen Fällen unpolaren Lösungsmitteln bestimmt. da der wahrscheinlichste Mechanismus dieser Reaktionen (siehe unten) höchstens eine sehr geringe Abhängigkeit der Aktivierungsparameter von der Lösungsmittelpolarität erwarten läßt, ist der direkte Vergleich aller Werte sicherlich eine gute Näherung.

Im Vergleich zu früher ${ }^{[17 a]}$ ergaben die jetzigen Messungen der 7-Umlagerung mit $E_{\mathrm{a}}=37.6 \mathrm{kcal} \mathrm{mol}^{-1}$ einen um 1.2 $\mathrm{kcal} \mathrm{mol}{ }^{-1}$ kleineren Wert. Zur Gegenüberstellung der Reaktivität von 7 und seinen Derivaten haben wir relative Geschwindigkeitskonstanten $\left(k_{\mathrm{rcl}}\right.$ in Tab. 1) berechnet und die von $7 \equiv 1$ gesetzt. Als Temperatur hierfür wählten wir $150^{\circ} \mathrm{C}$, weil dieser Wert nahe am oder im Meßbereich der Mehrzahl der Substrate liegt. Lediglich 7, 17 und 20 erforderten Extrapolationen über $50-60^{\circ} \mathrm{C}$. Die $\Delta S^{\ddagger}$ - und $\lg A-$ Werte zeigen große Schwankungen $\left(+5 \mathrm{bis}-14 \mathrm{cal} \mathrm{mol}^{-1}\right.$ $\mathrm{K}^{-1}$ bzw. 10.4-14.5), die auf der Basis des wahrscheinlichsten Mechanismus nicht einsichtig erscheinen. Ursache dafür sind relativ große Unsicherheiten bei den Geschwindigkeitskonstanten, besonders dort, wo Nebenreaktionen eine Rolle spielen und/oder nur bei zwei Temperaturen gemessen wurde. Die angegebenen Standardabweichungen ermittelten wir ohne die Unsicherheiten der $k$-Werte, weshalb die tatsächliche Fehlerbreite in den genannten kritischen Fällen erheblich größer ist. Die Diskussion stützt sich daher im wesentlichen auf die $k_{\text {rcl }}$-Werte und nicht auf die Aktivierungsparameter. Die Gesamtheit der $\Delta S^{*}$ - und $\lg A$-Werte vermittelt den Eindruck, daß diese Größen wohl generell in der Nähe von $0 \mathrm{cal} \mathrm{mol}^{-1} \mathrm{~K}^{-1}$ bzw. 13 liegen.

Homobenzvalen (12) lagert wesentlich schneller um als sein Dihydroderivat $7^{[17 a]}$. Der jetzt ermittelte Faktor der Beschleunigung beträgt 3860. Auch in 3-Methylentricy- clo[4.1.0.0 $\left.0^{2,7}\right]$ heptan (15) ist die Ethylen-Einheit in Konjugation mit dem Bicyclobutan-System und erhöht die Reaktionsgeschwindigkeit im Vergleich zu 7 auf das 590fache ${ }^{[19]}$. Es ist deshaib nicht verwunderlich, daß 2Phenyltricyclo[4.1.0.0 $0^{2,7}$ heptan (22) $70 \mathrm{mal}$ so schnell umlagert wie die Stammsubstanz 7. Zwar ist dieser Faktor aus den erwähnten Gründen recht unzuverlässig, jedoch weist schon die im Vergleich zur 7-Umlagerung um $70^{\circ} \mathrm{C}$ erniedrigte Temperatur auf eine massive Beschleunigung.

Ein entscheidendes Experiment war nun der Einsatz des 1-Phenylderivats 20 von 7 . Hätten sich hierbei ähnliche Geschwindigkeitskonstanten wie bei 22 ergeben, wäre die $\mathrm{Hy}$ pothese von einem intermediären Diradikal des Typs 8 stark gestützt worden. Jedoch erfolgt der Übergang $20 \rightarrow 21 \mathrm{mit}$ praktisch der gleichen Geschwindigkeit wie $7 \rightarrow 11$. Es besteht somit das Phänomen, daß eine der zu lösenden Bindungen des Tricyclo[4.1.0.0 $\left.0^{2,7}\right]$ heptan-Systems durch eine Phenylgruppe an C-2 aktiviert wird, nicht aber durch eine solche an C-1. Dies spricht gegen eine Diradikal-Zwischenstufe des Typs 8, denn sie müßte durch eine Phenylgruppe an jedem der beiden Radikalzentren eine Stabilisierung erfahren.

Die Umlagerung des Tetracyclooctans 17 ist gegenüber der von 7 ein wenig verlangsamt. Dies überrascht nicht, da eine Cyclopropan-Einheit im Falle der zur Diskussion stehenden Mechanismen keinen großen Einfluß erwarten läßt. So stabilisiert sie ein nachbarständiges Radikalzentrum nur wenig ${ }^{[37 c]}$, und auch hinsichtlich der Absenkung der Energie des Úbergangszustands pericyclischer Reaktionen, an denen sich das nachbarständige C-Atom beteiligt, ist nichts Auffälliges bekannt. Damit ergibt sich lediglich die von vornherein kaum bezweifelte Aussage, daß eine zwitterionische Zwischenstufe, in der der augenscheinlich unbeteiligte Dreiring ein kationisches Zentrum stabilisieren würde, nicht auftritt.

1,2,3,4-Tetrahydro-1,2,3-methenonapthalin (25) lagert $410 \mathrm{mal}$ so schnell um wie 7. Somit hat die Benzoanellierung in Konjugation mit dem Bicyclobutan-System eine ähnliche Beschleunigung zur Folge wie die Ethylen-Einheiten in $\mathbf{1 2}$ und 15 und die Phenylgruppe in 22. Bemerkenswert ist der Befund, daß 25 und seine Phenylderivate 27, 32 und 35 ähnlich schnell reagieren. Die relativen Geschwindigkeitskonstanten der reaktivsten Verbindung (35) und der am wenigsten reaktiven (27) unterscheidet lediglich ein Faktor von ca. 11. Verglichen mit 25 geht das 1-Phenylderivat 35 siebenmal so schnell in den Cyclobuten-Abkömmling über. Die Gegenwart eines zweiten Arylsubstituenten an einem seitli-

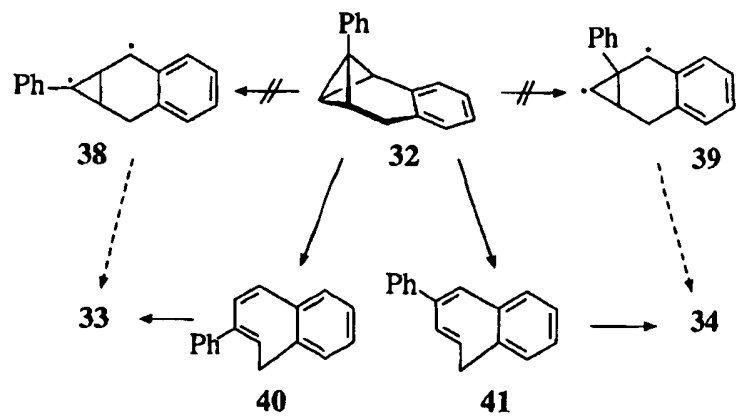

Chem. Ber. 1992, 125, 485-497 
chen C-Atom des Bicyclobutan-Systems steigert zwar die Geschwindigkeitskonstante weiter, aber nur um einen Bruchteil des Faktors der ersten Arylsubstituenten.

Im Hinblick auf ein Diradikal des Typs 8 als Zwischenstufe liefert die Thermolyse des Phenylderivats 32 von 25 ebenso wie schon 20 eine eindeutig negative Antwort. In 32 tragen beide C-Atome einer der Bindungen, die bei der Umlagerung gespalten werden kann, Arylgruppen. Dies sollte bei der geschwindigkeitsbestimmenden Bildung einer Diradikal-Zwischenstufe zu einem Vorzug von ca. $11 \mathrm{kcal}$ $\mathrm{mol}^{-1}{ }^{[18]}$ zugunsten von $38 \mathrm{im}$ Vergleich zur Alternative 39 führen. Damit würde nur das Folgeprodukt von 38, d.h. 33, entstehen. Abgesehen davon, daß die selektive Umwandlung $38 \rightarrow 33$ ohne den Konkurrenzprozeß $38 \rightarrow 7$-Phenyl-5Hbenzocyclohepten, wie schon diskutiert ${ }^{[15]}$, schwer verständlich wäre, entsteht aber aus 32 neben 33 in erheblichem Ausmaß 34 (33:34 = 82:18). Dies ist mit einem mechanistischen Schema, das sich auf die Diradikale 38 und 39 stützt, nicht in Einklang. Der konzertierte Übergang von 32 in Derivate von $(E, Z)-1,3-C y c l o h e p t a d i e n(10)$, etwa 40 und 41 oder ihre Isomere mit ausgetauschten Konfigurationen der Ethylen-Einheiten, erklärt den Befund besser, daß die durch zwei Arylgruppen aktivierte Bindung von 32 (C-1 - C2) nur ca. viermal so schnell gebrochen wird wie die einfach aktivierte (C-1 - C-9). Diese geringfügige Wirkung der Phenylgruppe in 32 bestätigt das schon bei 20 entdeckte Phänomen.

Die kinetischen Daten in Tab. 1 zeigen Parallelen zu den Werten der thermischen Umlagerung von Cyclobutenen

Tab. 4. Arrheniussche Aktivierungsparameter und relative Geschwindigkeitskonstanten bei $150^{\circ} \mathrm{C}$ der elektrocyclischen Ringöffnung von Cyclobutenen

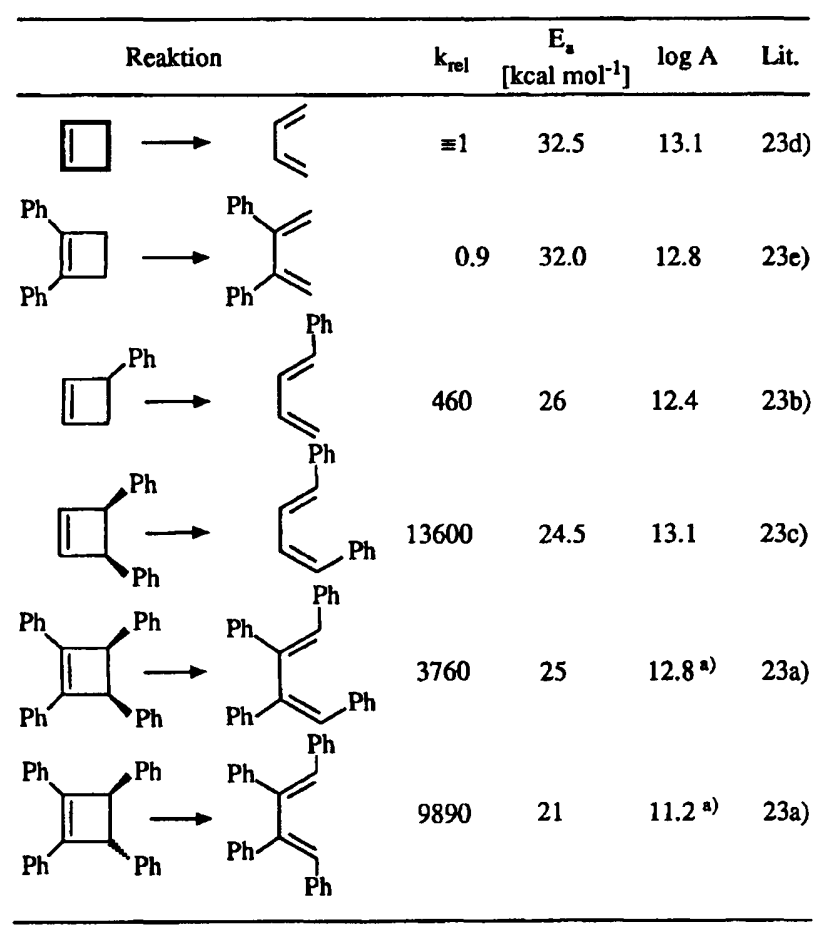

a) Aus Daten in Lit. ${ }^{\text {[23a] }}$ berechnet.
(Tab. 4), die als konzertierte Reaktion mit konrotatorischem Verlauf $\mathrm{zu}$ betrachten ist ${ }^{[4,38]}$ und wie die Umlagerung von Bicyclobutanen zu 1,3-Butadienen führt: erstens liegen die lg $A$-Werte im gleichen Bereich; zweitens wirkt eine Phenylgruppe an C-3 von Cyclobuten beachtlich beschleunigend $^{[23 a-d]}$ wie die Phenylgruppe in 22, die Benzogruppe in 25, die Naphthalin-Gruppierung in 16 und auch die EthylenEinheiten in 12 und 15, d.h. wie ein Aryl- oder Vinylsubstituent an C-2 des Bicyclobutan-Gerüsts; und drittens hat die Phenylgruppe an den olefinischen C-Atomen von Cyclobuten $^{[23 a, c-c]}$ wie eine Phenylgruppe an den Bicyclobutan-Brückenkopf-C-Atomen von 20 und 32, d. h. an C-1 des Bicyclobutan-Gerüsts, wenn überhaupt, nur einen sehr kleinen Effekt.

Die Wirkung einer Phenylgruppe an C-3 von Cyclobuten und $\mathrm{C}-2$ von Bicyclobutan kann damit beschrieben werden, $\mathrm{da}$ diese Substituenten im Zuge der Reaktion in Konjugation mit dem $\pi$-Elektronen-System des 1,3-Butadiens gelangen. Allerdings beläuft sich die thermochemische Konjugationsenergie zwischen Phenyl- und Vinylgruppe wie in Styrol nur auf $2.2 \mathrm{kcal} \mathrm{mol}^{-1}$ [39]. Damit ist im Úbergangszustand der Cyclobuten- und Bicyclobutan-Isomerisierung die Wirkung einer C-3- bzw. C-2-Phenylgruppe stärker als im Produkt. D.h. eine Phenylgruppe an einem C-Atom, an dem eine $\sigma$-Bindung gelöst und eine $\pi$-Bindung geknüpft wird, übt im Übergangszustand eine Stabilisierung aus, die größer ist als die neu gewonnene Mesomerie-Energie des Produkts. Diese Stabilisierung des Übergangszustands ist etwa halb so groß wie die auf ca. $11 \mathrm{kcal} \mathrm{mol}^{-1}{ }^{[18]}$ festleg. bare Mesomerie-Energie in einem Benzyl-Radikal.

1,2-Diphenyl-1-cyclobuten ${ }^{\text {[23e] }}$ lagert etwa gleich schnell um wie Cyclobuten ${ }^{[23 \mathrm{~d}]}$ selbst. Die Phenylgruppen im Ausgangsmaterial stehen wie im Produkt an $\mathrm{sp}^{2}$-hybridisierten C-Atomen. Dies führt zu keiner Stabilisierung des Übergangszustands durch die Substituenten. Auch die Phenylgruppen in $\mathbf{2 0}$ und 32, die an C-1 des Bicyclobutan-Gerüsts gebunden sind, haben kaum eine Wirkung auf die Geschwindigkeit der Umlagerung. Anders als in 1,2-Diphenyl1-cyclobuten wird aber in 20 und 32 eine $\sigma$-Bindung am phenyltragenden C-Atom gespalten und durch eine $\pi$-Bindung ersetzt. Der sehr kleine Effekt dieser Phenylgruppen bietet das entscheidende Argument gegen die Diradikalzwischenstufe. Warum jedoch der konzertierte Prozeß nicht wenigstens eine moderate, sondern bestenfalls eine kleine $\mathrm{Be}$ schleunigung erfährt (Faktor 1.1 bei 20 gegenüber 7, Faktor 4.3 bei 32 gegenüber 25), ist schwer verständlich. Uns ist auch kein Präzedenzfall bekannt. cis-3,4-Diphenyl-1-cyclobuten $^{[23 \mathrm{c}]}$ (Tab. 4) belegt im Vergleich zu 3-Phenyl-1-cyclobuten $^{[23 b]}$, daß je eine Phenylgruppe an beiden Enden der zu spaltenden $\sigma$-Bindung die Umlagerung wesentlich stärker beschleunigen (Faktor 13600) als insgesamt nur eine Phenylgruppe (Faktor 460), auch wenn ein Teil der Beschleunigung bei cis-3,4-Diphenyl-1-cyclobuten wohl auf die Destabilisierung des Grundzustands zurückzuführen ist. Im Beispiel 32 zur Bicyclobutan-Thermolyse trifft dies nicht zu. Möglicherweise wechselwirkt die Phenylgruppe an C-1 des Bicyclobutan-Gerüsts intensiv mit der Zentralbindung, die ja eine aus zwei fast reinen $p$-Orbitalen konstituierte $\sigma$-Bin- 
dung ist ${ }^{[5]}$. Auf dem Weg zum Übergangszustand müßte dann diese Konjugation zum Teil aufgegeben werden, was wohl die Stabilisierung, die die Phenylgruppe bei der Lösung der seitlichen Bicyclobutan-Bindung ausüben könnte, in etwa aufwiegt.

Ohne Zweifel schließen die bisher diskutierten Daten die Öffnung des Bicyclobutan-Systems zu einem Diradikal aus und stützen eine konzertierte Umlagerung in eine 1,3-Butadien-Einheit. Wie synchron ist aber dieser Prozeß? Vollzieht sich also die Lösung der beiden $\sigma$-Bindungen gleichzeitig in gleichem Maße, oder ist im Übergangszustand eine der beiden Bindungen stärker gestreckt als die andere? Zu dieser Frage bietet das Cyclobuten-Modell keine Parallele, jedoch geben die kinetischen Daten der Thermolyse von 27 eine klare Antwort. In 27 tragen C-2 und C-4 des Bicyclobutan-Gerüsts, d.h. die äußeren Termini der zu lösenden $\sigma$ Bindungen, je eine Arylgruppe. Somit liegt eine Situation vor, die die Aktivierung beider Bindungen erwarten läßt, wenn man sich auf die Modelle 22 und 25 beruft, die jeweils nur über eine Arylgruppe verfügen und durch diese im Vergleich zur Stammsubstanz 7 erheblich beschleunigt umlagern. Tatsächlich reagiert jedoch 27 etwas langsamer als 25 (Faktor 0.6) und nur wenig schneller als 22 (Faktor 3.7). Dieser Befund belegt, daß eine der Arylgruppen von 27 im wesentlichen unwirksam bleibt. Damit ist nur ein stark asynchroner pericyclischer Prozeß im Einklang, nicht aber ein synchroner.

\section{C.3. SchluBfolgerung}

Die Effekte der Arylgruppen am Tricyclo[4.1.0.0.7 $]$ heptan-System auf die Geschwindigkeitskonstanten schließen für die thermische Umlagerung sowohl einen Mechanismus mit der geschwindigkeitsbestimmenden Bildung eines Diradikals als auch einen synchronen konzertierten Verlauf aus. Der von Shevlin und McKee ${ }^{[9]}$ anhand quantenmechanischer Rechnungen vorgeschlagene unsymmetrische Übergangszustand 6, der einem einstufigen aber stark asynchronen Verlauf entspricht, erklärt die experimentellen $\mathrm{Er}$ gebnisse zum Übergang der Bicyclobutan- in eine 1,3Butadien-Einheit am besten, zumindest am Beispiel des Tricyclo[4.1.0.0 $\left.0^{2,7}\right]$ heptan-Systems.

Die Enthalpien der Übergangszustände der Umlagerungen $32 \rightarrow 33$ und $35 \rightarrow 36$ liegen knapp $30 \mathrm{kcal} \mathrm{mol}^{-1}$ über denen der Ausgangsverbindungen. Geht man davon aus, daß das Diradikal $549 \mathrm{kcal} \mathrm{mol}^{-1}$ energiereicher ist als Bicyclobutan $(1)^{[9,15]}$ und daß dies in etwa auch für 8 relativ zu 7 gilt, so führt die zweimalige Subtraktion der Benzylradikal-Mesomerieenergie (ca. $11 \mathrm{kcal} \mathrm{mol}^{-1}{ }^{[18]}$ ) $\mathrm{zu} 27 \mathrm{kcal}$ $\mathrm{mol}^{-1}$ als Schätzwert der Reaktionsenthalpien für $32 \rightarrow \mathrm{Di}$ radikal 38 und die analoge Bildung eines Diradikals aus $\mathbf{3 5}$, wobei im letzteren Fall die Additivität der Wirkungen zweier Arylgruppen auf ein Radikalzentrum vorausgesetzt ist. Bei Gültigkeit dieser Schätzung sollten also 32 und 35 den Umlagerungsweg über ein Diradikal bevorzugen. Wie oben geschildert, sprechen aber die experimentellen Befunde gegen diesen Verlauf und charakterisieren damit die Abschätzung der Enthalpien von 38 und des analogen, zu 35 gehörenden Diradikals als zu grobe Näherung. Insbesondere könnte bei
38 eine Rolle spielen, daß sich die Benzylresonanz-Stabilisierung im Falle der Phenylcyclopropyl-Einheit nicht voll entfalten kann, weil die Einebnung des Radikalzentrums im Dreiring zur Erhöhung der Spannungsenergie führt.

Vom Úbergangszustand 6 aus soll 1,3-Butadien mit einem Torsionswinkel von $90^{\circ}$ zwischen beiden Vinylgruppen entstehen $^{[9]}$. Im Falle der Umlagerung von 7 und seinen Derivaten ist von einer $(E, Z)$-1,4-disubstituierten Butadien-Einheit auszugehen, die in einen Siebenring inkorporiert ist, z. B. $10^{[15]}, 13,40$ und 41. Hier paßt ein Torsionswinkel von $90^{\circ}$ gut zur günstigsten Konformation, weil die $s$-cis oder $s$ trans-Form wegen zu hoher Spannungsenergien unerreichbar sind, und für den konrotatorischen Ringschluß zu Bicyclo[3.2.0]hept-6-en (11) und seinen Derivaten ist diese $90^{\circ}$ Konformation eine vorteilhafte Voraussetzung.

Azulvalen ${ }^{[40 a]}$ und Heptalvalencarbonitril ${ }^{[406]}$, die Derivate von Homobenzvalen (12) sind, gehen mit $E_{\mathrm{a}}=28.6 \mathrm{kcal}$ $\mathrm{mol}^{-1}$ und $\lg A=12.3$ bzw. $E_{\mathrm{a}}=27.2 \mathrm{kcal} \mathrm{mol}^{-1}$ und $\mathrm{lg}$ $A=12.7$ ohne Einschaltung eines Cyclobuten-Derivats in Azulen bzw. das entsprechende Heptalencarbonitril über. Bei Azulvalen kann die Einstufigkeit als Zehn-ElektronenProze $B$ interpretiert werden ${ }^{[40 a]}$ in Analogie zur Umlagerung von Benzvalen in Benzol, die man als konzertierten SechsElektronen-Prozeß betrachtet ${ }^{[40 \mathrm{c}]}$. Dagegen paßt das Verhalten des Heptalvalen-Derivats ${ }^{(40 \mathrm{~b}]}$ weder in das eine, hier gewonnene Bild noch in jenes von der Thermolyse der Aromaten-Valene.

Der Deutschen Forschungsgemeinschaft und dem Fonds der chemischen Industrie danken wir für die Förderung.

\section{Experimenteller Teil}

Geräte: Lit. ${ }^{[21 a]}$ - Zur Konzentrationsermittlung bei den kinetischen Messungen dienten folgende NMR-Spektrometer: Varian T 60 (Verbindung 7), Varian EM $360(17,25)$, Varian EM 390 (20, 22, 35), Bruker AC $200(27,32,35)$

Kinetische Messungen: Alle bei den Thermolysen verwendeten Substanzen und Lösungsmittel wurden nach den üblichen Verfahren soweit wie möglich gereinigt und getrocknet. Nach Einbringen der Probenlösung in ein dickwandiges NMR-Röhrchen entgaste man dic Lösung durch mehrere Einfrieren-Abpumpen-AuftauenZyklen, schmolz das Röhrchen i. Vak. ab und thermolysierte die Probe durch vollständiges Eintauchen des Röhrchens in ein thermostatisiertes Bad (Siliconöl, Lauda-NBS/S15/22-Thermostat, $\pm 0.1^{\circ} \mathrm{C}$ ). In geeigneten Abständen entnahm man die Probe, führte NMR-analytisch die Gehaltsbestimmung durch und thermolysierte dann weiter. Pro Einzelversuch wurde die Konzentration ohne Anfangs- und Endpunkt an 6-14 Zeitpunkten ermittelt. Den Endwert erhielt man durch weiteres Erhitzen über den letzten Meßpunkt hinaus, meist bei noch etwas erhöhter Temperatur, bis keine Ausgangsverbindung mehr beobachtbar war. Zur NMR-analytischen Gehaltsbestimmung dienten entweder die Integrale oder die gedehnten Banden, deren Flächen man durch Ausschneiden und Wägen ermittelte, freiliegender Signale von Ausgangsverbindung und/oder Produkt. Mit Hilfe der so erhaltenen Konzentrationen wurden die Geschwindigkeitskonstanten nach 1. Ordnung berechnet. Diese finden sich zusammen mit den Aktivierungsparametern, die aus den Geschwindigkeitskonstanten bei verschiedenen Temperaturen nach den Gleichungen von Arrhenius und Eyring hervorgingen, in Tab. 1 
Thermolyse von Tricyclo[4.1.0.0 $\left.0^{2,7}\right]$ heptan (7): Ca. $100 \mathrm{mg} 7^{[13,41 \mathrm{a}]}$ wurden mit $75 \mathrm{mg}$ Mesitylen (interner Standard) und $30 \mathrm{mg}$ $N, N, N^{\prime}, N^{\prime}$-Tetramethylethylendiamin (TMEDA) in $0.3 \mathrm{ml}\left[\mathrm{D}_{8}\right]$ Toluol gemischt. Man fügte eine Spatelspitze $\mathrm{LiAlH}_{4}$ zu, beließ die Mischung $15 \mathrm{~h}$ bei $20^{\circ} \mathrm{C}$, verdampfte dann die flüchtigen Stoffe i. Vak. und kondensierte sie in einem mit flüssigem Stickstoff gekühlten NMR-Röhrchen, das zuvor bei angelegtem Hochvakuum mit einer offenen Flamme ausgeheizt worden war. Die Thermolysen wurden bei 199.7 und $220.1^{\circ} \mathrm{C}$ ausgeführt (siehe Tab. 1). Nach dre Halbwertszeiten waren Bicyclo[3.2.0]hept-6-en (11) und 1,3-Cycloheptadien (9) bei praktisch quantitativer Ausbeute im Verhältnis 98.8:1.2 entstanden. Beim Endpunkt, d. h. bei so gut wie vollständig umgesetztem 7, lag der Gehalt an 9 bei mehreren Prozent. Die Konzentrationsbestimmungen erfolgten anhand des Pseudotripletts von 11 bei $\delta=5.93$, der breiten Bande von 9 bei 5.85 und des Mesitylen-Signals bei 6.80 .

Versuche zur Ubertragung der Reaktion in den präparativen Maßstab schlugen fehl. Auch bei gleicher Probenvorbereitung entstanden beim Einsatz von $1 \mathrm{~g} 7 \mathrm{im}$ Bombenrohr immer signifikante Mengen an 2-Norcaren.

Als TMEDA als Komponente des Thermolysegemisches weggelassen wurde, entstand bei $200^{\circ} \mathrm{C}$ aus 7 ein 1.4:1.0-Gemisch aus 11 und 3-Methylen-1-cyclohexen. Die letztere Verbindung wurde durch Vergleich ihres 'H-NMR-Spektrums (in $\left[D_{8}\right]$ Toluol) mit dem der nach Gassman und Atkins ${ }^{[24]}$ bereiteten authentischen Substanz identifiziert: $\delta=1.4-2.5\left(\mathrm{~m} ; 4,5,6-\mathrm{H}_{2}\right), 4.86$ (br. s; $\left.=\mathrm{CH}_{2}\right), 5.78$ und 6.25 (jeweils dm, $J_{1,2} \approx 10.5 \mathrm{~Hz} ; 1,2-\mathrm{H}$ ). - ${ }^{1} \mathrm{H}-\mathrm{NMR}$ von 11: Tab. 2. $-{ }^{13} \mathrm{C}-\mathrm{NMR}$ von 11: Tab. 3.

Thermolyse von Tetracyclo[5.1.0.0.2. $.0^{3.5}$ Joctan (17) und seinem Dideuterioderivat 17a: Die Synthese von 17 kann entweder aus Benzvalen via Homobenzvalen (12) ${ }^{[25]}$ oder ökonomischer aus 1,4Cyclohexadien ${ }^{[26]}$ erfolgen. - Für die kinetischen Untersuchungen wurden die Proben wie bei der Thermolyse von 7 vorbereitet. Sie erfolgten bei 209.3 und $229.3^{\circ} \mathrm{C}$ (siehe Tab. 1) durch Messung der Abnahme des 17-Gehalts anhand des Quintetts bei $\delta=2.74$, der Zunahme des Gehalts an Tricyclo[4.2.0.0 2.4 $]$ oct-7-en (18) anhand des breiten Singuletts bei 6.17 (bei $60 \mathrm{MHz}$; 7,8-H) und des Quartetts bei $-0.21\left(J_{2,3 \alpha} \approx J_{3,3} \approx J_{3 \alpha, 4} \approx 4.3 \mathrm{~Hz} ; 3 \alpha-\mathrm{H}\right)$ und der $\mathrm{Zu}$ nahme des Gehalts an Bicyclo[5.1.0 Jocta-2,4-dien (19) anhand der olefinischen Signale bei 5.5-6.1 jeweils unter Normierung auf das Intcgral des Mesitylen-Signals bei 6.80. Bei quantitativer Ausbeute lag nach einer Halbwertszeit das Verhältnis 18:19 bei ca. 87:13, nach zwei Halbwertszeiten bei ca. 77:23. Nach drei Halbwertszeiten deutete sich Bildung einer weiteren Verbindung an durch ein Pseudotriplett bei $\delta=6.44$ (Linienabstand $4.2 \mathrm{~Hz}$ ) und ein Quartett bei $-0.58(J=4.3 \mathrm{~Hz})$. Bei weiterem Erhitzen (mehr als zehn Halbwertszeiten) wurde 18 vollständig zugunsten dieses neuen Produkts verbraucht. Vermutlich handelt es sich um das Diels-Alder-Addukt von 18 an das Diensystem von 19. Daneben war eine kleine Menge Benzol entstanden, wohl als Zerfallsprodukt von $19^{[29]}$.

17a gewannen wir durch Behandlung von $17 \mathrm{mit} n$-Butyllithium und Reaktion des lithiierten 17 mit $\mathrm{D}_{2} \mathrm{O}$ nach der Vorschrift für $\left[3,4-\mathrm{D}_{2}\right]$ Tetracyclo[4.1.0.0 $\left.0^{2.4} .0^{3.5}\right]$ heptan $^{[41 \mathrm{~b}]}$. Im ${ }^{1} \mathrm{H}-\mathrm{NMR}-\mathrm{Spek}-$ trum $\left(\mathrm{C}_{6} \mathrm{D}_{6}\right)$ von 17 a fehlt im Vergleich zu dem von $17^{[26]}$ das bei $\delta=1.39$ zentrierte Multiplett und die Feinstruktur der Banden bei 2.74 und 2.16. - Zur Thermolyse von 17a bereiteten wir die Probe ähnlich wie bei 17 vor; wir verwendeten lediglich $\left[D_{6}\right]$ Benzol statt $\left[D_{8}\right]$ Toluol. Daß sich nach $6 \mathrm{~h}$ bei $210^{\circ} \mathrm{C}$ etwa die Hälfte von $17 \mathrm{a}$ in $\left[7,8-D_{2}\right]$ Tricyclo[4.2.0.0 $\left.{ }^{2.4}\right]$ oct-7-en (18 a) umgewandelt hatte, bewies das 'H-NMR-Spektrum durch das Quartett bei $\delta=-0.21$ und die weitgehende Abwesenheit des breiten Singuletts bei 6.17 . Das Restsignal geht auf den unvollständigen Deuteriumgehalt von 17 a zurück.
Thermolyse von 1-Phenyltricyclo[4.1.0.0 $0^{2.7}$ ]heptan (20): Man löste $180 \mathrm{mg} \mathrm{20} 0^{[21 \mathrm{a}, 31]}, 20 \mathrm{mg}$ Cyclohexen (interner Standard) und $40 \mathrm{mg}$ Cyclohexan (Locksignal für das NMR-Spektrometer) in $0.2 \mathrm{ml}$ $\mathrm{C}_{6} \mathrm{D}_{6}$, versetzte das Gemisch mit einigen Spatelspitzen $\mathrm{LiAlH}_{4}$ und bewahrte es über Nacht bei $20^{\circ} \mathrm{C}$ auf. Dann dampfte man das Gemisch in einer sorgfältig getrockneten Apparatur (Erhitzen mit einer Flamme bei angelegtem Hochvakuum) bei $20^{\circ} \mathrm{C} / 10^{-4}$ Torr vom $\mathrm{LiAlH}_{4} \mathrm{ab}$, kondensierte es nach sehr kurzem Weg in einem gekühlten Kölbchen mit angeschmolzenem NMR-Röhrchen, fülite es durch Drehen des Kölbchens in das NMR-Röhrchen und schmolz dieses i. Vak. ab. Die Thermolysen erfolgten bei 197.4 und $219.5^{\circ} \mathrm{C}$. Der zeitliche Verlauf wurde anhand der Abnahme der Intensität des 2,6-H-Signals von 20 bei $\delta=2.73$ und der Zunahme der Intensität des 7-H-Tripletts von 6-Phenylbicyclo[3.2.0] hept-6en (21) bei 5.94 verfolgt, wobei das Cyclohexen-Signal bei 5.60 als interner Standard diente. Bei Umsätzen von $80-91 \%$ lagen die Ausbeuten von 21 , bezogen auf umgesetztes 20, bei $48-82 \%$. Vom nicht identifizierten zweiten Produkt stammen breite, bei $\delta=5.70$ und 6.05 zentrierte Banden.

Zur Isolierung von 21 thermolysierte man mehrere Proben nach Messung der Kinetik, bis kein 20 mehr beobachtbar war, engte dann die vereinigten Lösungen bei $20^{\circ} \mathrm{C} / 20$ Torr ein und erhielt aus dem Rückstand durch Destillation bei $70^{\circ} \mathrm{C}(\mathrm{Bad}) / 0.1$ Torr ein farbloses $\mathrm{OI}$, das durch Dickschichtchromatographie (Kieselgel, $n$ Hexan) gereinigt wurde. Erneute Destillation bei $70^{\circ} \mathrm{C}(\mathrm{Bad}) / 0.1$ Torr lieferte reines 21. Die Reinigungsoperationen gingen mit groBen Substanzverlusten einher. - ${ }^{1}$ H-NMR: Tab. $2 .-{ }^{13} \mathrm{C}$-NMR: Tab. 3.

Thermolyse von 2-Phenyltricyclo[4.1.0.(1) ${ }^{2.7}$ ]heptan (22): Man löste ca. $200 \mathrm{mg}$ eines $31: 8: 3: 1-\mathrm{Gemischs}^{[21 \mathrm{a}]}$ aus 22, 3-Phenyltricyclo[4.1.0.0.0.7. Phenylbicyclo[3.2.0]hept-6-en (23), $40 \mathrm{mg}$ Cyclohexan, $30 \mathrm{mg}$ TMEDA und $10 \mathrm{mg}$ Anisol (interner Standard) in $0.2 \mathrm{ml} \mathrm{C}_{6} \mathrm{D}_{6}$, trocknete das Gemisch mit $\mathrm{LiAlH}_{4}$ und brachte es, wie bei der Thermolyse von 20 beschrieben, ins NMR-Röhrchen. Die Reaktionen wurden bei $129.9,130.0,137.9$ und $148.0^{\circ} \mathrm{C}$ ausgeführt. Den zeitlichen Verlauf verfolgte man anhand der Zunahme der Intensität der Doppeldubletts von 6- und 7-H von 1-Phenylbicyclo [3.2.0 /hept6 -en (23) bei $\delta=5.94$ und 6.04 sowie des 3-H-Multipletts von 2Phenyl-2-norcaren (24) bei 5.64 (vgl. Lit. ${ }^{[21 a]}$ ), wobei das MethoxySingulett von Anisol bei 3.42 als interner Standard diente. Von 22 lag keine Bande frei, so daß die Bestimmung seiner Anfangskonzentration nicht möglich war. Da über 23 und 24 hinaus kein Produkt gefunden wurde, nehmen wir an, daß der Übergang von 22 in 23 und 24 quantitativ erfolgte. Das Verhältnis 23:24 war bei den einzelnen Versuchen zeitlich nicht konstant; es belief sich kurz nach Beginn der Thermolysen auf 1.17-0.66 und am Ende auf 0.90 bis 0.47 .

Zur Isolierung von 23 thermolysierte man mehrere Proben nach Messung der Kinetik, bis die Konzentrationen von 23 und 24 nicht mehr zunahmen, engte die vereinigten Lösungen bei $20^{\circ} \mathrm{C} / 20$ Torr ein, destillierte die flüchtigen Anteile bei $70^{\circ} \mathrm{C}(\mathrm{Bad}) / 0.1$ Torr aus dem Rückstand und gewann reines 23 durch präparative Gaschromatographie (Säule: $6 \mathrm{~m}, 25 \%$ Silicon SE 30 auf Chromosorb ${ }^{n} \mathrm{~W} /$ AW DMCS, $130^{\circ} \mathrm{C}$ ). - 'H-NMR. Tab. 2. -- ${ }^{13} \mathrm{C}-\mathrm{NMR}$ : Tab. 3.

\section{$\mathrm{C}_{13} \mathrm{H}_{14}$ (170.3) Ber. C 91.71 H 8.29 Gef. C 91.61 H 8.31}

Thermolyse von 1,2,3,4-Tetrahydro-1,2,3-methenonaphthalin (25):

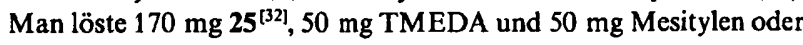
$30 \mathrm{mg}$ Cyclohexan (interner Standard) in $0.3 \mathrm{ml} \mathrm{C}_{6} \mathrm{D}_{6}$, trocknete das Gemisch mit $\mathrm{LiAlH}_{4}$ und verfolgte die Reaktion bei $135.8-160.2^{\circ} \mathrm{C}$ wic üblich anhand der Abnahme der Intensität des 2,9-H-Tripletts von 25 bei $\delta=1.98$ und der Zunahme der Intensität 
der 1 - und 2-H-Signale von 3,7b-Dihydro-2aH-cyclobut/a/inden $(26)^{[42]}$ bei 6.10 und 6.33. Die Bildung von 26 aus 25 verlief ohne Nebenprodukte.

Zur Isolierung von 26 erhitzte man mehrere Proben nach Messung der Kinetik bis zum vollständigen Umsatz von 25 und gewann reines 26 durch präparative Gaschromatographie (Säule wie bei der

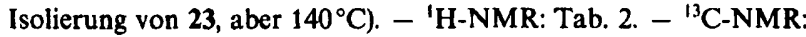
Tab. 3.

Thermolyse von 1,2,3,4-Tetrahydro-3-phenyl-1,2,3-methenonaphthalin (27): Man rührte Gemische aus $1 \mathrm{ml} \mathrm{C}_{6} \mathrm{D}_{6}, 0.3 \mathrm{ml}$ TMEDA und $0.04 \mathrm{ml}$ Cyclohexen oder $0.1 \mathrm{ml} \mathrm{C}_{6} \mathrm{D}_{6}, 1.5 \mathrm{ml}$ TMEDA und $0.06 \mathrm{ml}$ Cyclohexen $1 \mathrm{~h}$ mit einer Spatelspitze $\mathrm{LiAlH}_{4}$, entgaste sie dann durch drei Einfrieren-Abpumpen-Auftauen-Zyklen, verdampfte die flüchtigen Anteile i. Vak. und kondensierte sie in einem mit flüssigem Stickstoff gekühlten NMR-Röhrchen, das zuvor mit ca. $20 \mathrm{mg}$ reinem und trockenem $27^{[21 \mathrm{a}]}$ beschickt worden war. Nach Abschmelzen des NMR-Röhrchens i. Vak. wurde bei 137.5 und bei $156.0^{\circ} \mathrm{C}$ (jeweils zwei Versuche) thermolysiert. Die Anfangskonzentration von 27 ermittelte man durch Integration des $4-\mathrm{H}_{2}$-Singuletts bei $\delta=2.97$ im Vergleich zum Integral des Cyclohexen-Signals bei 5.69. Den zeitlichen Verlauf kontrollierte man anhand der Intensität der 7-H-Dubletts von 6-Phenyl-5H-benzocyclohepten (29) und 8Phenyl-5H-benzocyclohepten (31) sowie der 1 - und 2-H-Doppeldubletts von 3,7b-Dihydro-2a-phenyl-2aH-cyclobut/a]inden (28), die nahe benachbart bei $\delta \approx 6.23$ auftauchten. Als $\mathrm{MaB}$ für $1 a, 7 b$ Dihydro-2-phenyl-1H-cyclopropa[a]naphthalin (30) diente das In-

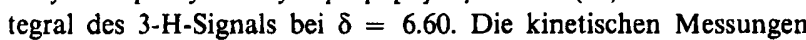
wurden über 2.6-3.4 Halbwertszeiten ausgeführt. Zur Bestimmung der Endkonzentrationen wurden die Proben solange erhitzt, bis eine Zunahme der Signalintensitäten von 29-31 nicht mehr beobachtbar war. Die Ausbeuten, bezogen auf eingesetztes 27, betrugen über $90 \%$, und zwar für $2959-70 \%$, für $317-22 \%$ und für 30 $11-23 \%$.

Aus den Konzentrationen von 28, 29 und 31 im Verlauf der Reaktion folgt, daß die Verbindungen auseinander hervorgehen. So lag bei einem Versuch nach 0.18 Halbwertszeiten das Verhältnis 28: 29 bei 1.0:2.6, während die Signale von 31 noch nicht erkennbar waren. Nach 1.02 Halbwertszeiten war 28:29 = 1.0:5.7, und die Signale von 31 konnte man bestenfalls erahnen. Dagegen fand man nach 3.29 Halbwertszeiten die Banden von 28 nicht mehr, und 29:31 lag bei 4.2:1.0. Als Beleg für die Struktur von 28, das nur intermediär beobachtet wurde, dienen die ${ }^{1} H-N M R-S i g n a l e$ (Tab. 2) der nichtaromatischen Strukturelemente.

Versuche zur Trennung von 29-31 durch Mitteldruckchromatographie $\left(\mathrm{SiO}_{2}\right.$, Petroleumbenzin/Essigester 95:5) scheiterten.

29: ${ }^{1} \mathrm{H}-\mathrm{NMR}\left(\mathrm{CDCl}_{3}\right): \delta=3.51\left(\mathrm{~s} ; 5-\mathrm{H}_{2}\right), 6.37\left(\mathrm{~d}, J_{7,8}=5.9 \mathrm{~Hz}\right.$; 7-H), 6.61 (dd, $\left.J_{8,9}=11.4 \mathrm{~Hz} ; 8-\mathrm{H}\right), 7.12(\mathrm{~d} ; 9-\mathrm{H}), 7.18-7.46(\mathrm{~m} ; 7$ aromat. $\mathrm{H}), 7.55(\mathrm{~m} ; 2$ aromat. $\mathrm{H}) .-{ }^{13} \mathrm{C}-\mathrm{NMR}\left(\mathrm{CDCl}_{3}\right): \delta=38.1$ (t; C-5), 122.1, 125.7, 127.2, 127.4, 127.7, 128.8, 129.0, 132.8 (jeweils d; C-1,2,3,4,7,8,9, p-C), 126.7 (d; $o-C), 128.4$ (d; $m-\mathrm{C}), 135.8,136.2$, 138.7, 141.6 (jeweils s; C-4a,6,9a, ipso-C).

31: ${ }^{1} \mathrm{H}-\mathrm{NMR}\left(\mathrm{CDCl}_{3}\right): \delta=3.10\left(\mathrm{~d}, J_{5,6}=7.0 \mathrm{~Hz} ; 5-\mathrm{H}_{2}\right), 5.98(\mathrm{dt}$, $J_{6,7}=10.0 \mathrm{~Hz} ; 6-\mathrm{H}$ ), 6.30 (br. d; $7-\mathrm{H}$ ); die Signale der aromat. $\mathrm{H}$ und von $9-H$ sind von denen der aromat. $H$ von 29 und 30 überlagert.

Behandlung von 27 mit Tetracyanethylen sowie Trifluoressigsäure: $\mathrm{Zu}$ einer Lösung von $100 \mathrm{mg}(0.46 \mathrm{mmol}) 27$ in $5 \mathrm{ml}$ Acetonitril gab man unter Rühren bei $20^{\circ} \mathrm{C} 59 \mathrm{mg}$ (0.46 mmol) Tetracyanethylen, wobei sofort eine grüne Färbung auftrat. Man ließ $45 \mathrm{~min}$ weiterrühren, engte dann i. Vak. ein, digerierte den zurückbleibenden grünen Feststoff mit $5 \mathrm{ml}$ Ether, filtrierte und führte mit dem eingeengten Filtrat eine Mitteldruckchromatographie durch $\left(\mathrm{SiO}_{2}\right.$,
Petroleumbenzin/Essigester 95:5), die ein 11:1-Gemisch aus $\mathbf{3 0}$ und 29 erbrachte.

In ein NMR-Röhrchen mit 27 in $\mathrm{CDCl}_{3}$ gab man einen Tropfen Trifluoressigsäure, was zur leichten Erwärmung der Probe führte. Das ${ }^{1} \mathrm{H}$-NMR-Spektrum zeigte die Bildung von 30 und wenig 29 an.

30: ${ }^{1} \mathrm{H}-\mathrm{NMR}\left(\mathrm{CDCl}_{3}\right): \delta=-0.07\left(\approx \mathrm{td}, J_{1,1}=3.6, J_{1 \text { endo, 1a }}=\right.$ $4.9, J_{\text {lendo,7b }}=5.2 \mathrm{~Hz}$; endo-1-H $), 1.72\left(\approx \mathrm{td}, J_{\text {lexo, } 1 \mathrm{a}}=8.8, J_{1 \text { exo,7b }}=\right.$ $9.0 \mathrm{~Hz} ;$ exo-1-H), 2.26 (dddd, $\left.J_{1 \mathrm{a}, 7 \mathrm{~b}}=7.8, J_{1 \mathrm{a}, 3}=0.8 \mathrm{~Hz} ; 1 \mathrm{a}-\mathrm{H}\right), 2.54$ (ddd; 7b-H), 6.64 (br. s; 3-H), $7.12-7.48$ (m; 7 aromat. H), 7.70 (m; 2 aromat. $\mathrm{H}) .-{ }^{13} \mathrm{C}-\mathrm{NMR}\left(\mathrm{CDCl}_{3}\right): \delta=9.3(\mathrm{t} ; \mathrm{C}-1), 18.3,20.7$ (jeweils d; C-1a,7b), 119.7 (d; C-3), 125.8, 127.0, 127.6, 127.9, 128.1 (jeweils d; C-4,5,6,7, p-C), 125.9 (d; $o-\mathrm{C}), 128.4$ (d; $m-\mathrm{C}$ ), 131.2, 134.9, 138.8, 140.4 (jeweils s; C-2,3a,7a, ipso-C).

Thermolyse von 1,2,3,4-Tetrahydro-2-phenyl-1,2,3-methenonaphthalin (32): Die Probenvorbereitung geschah wie bei der Thermolyse von 27. Die Anfangskonzentration ermittelte man ${ }^{i} \mathrm{H}-\mathrm{NMR}$-spektroskopisch anhand des Integrals des Doppeldoppeldubletts von $32^{[21 \text { a }]}$ bei $\delta=3.21$ im Vergleich zum Integral des CyclohexenSignals bei 5.60. Je zwei Proben wurden bei 136.3 und $152.9^{\circ} \mathrm{C}$ umlagert. Der Fortgang der Reaktion wurde durch Integration des Singuletts von 3,7b-Dihydro-2-phenyl-2aH-cyclobut/a]inden (33) bei $\delta=6.32$ und des Tripletts von 3,7b-Dihydro-1-phenyl-2aH-cyclobut [a Jinden (34) bei 6.03 über 3.6-4.2 Halbwertszeiten überwacht. Bei der Endwertsbestimmung lagen die Ausbeuten an 33 und 34 bei $74-81$ bzw. $16-18 \%$.

Das Gemisch aus 33 und 34 überstand die Chromatographie an Kieselgel nicht. Daher isolierte man es durch vollständiges Einengen der Proben der Geschwindigkeitsmessung i.Vak. NMR-Spektren (Tab. 2 und 3) und Elementaranalyse belegen die Reinheit dieses Gemisches.

\section{$\mathrm{C}_{17} \mathrm{H}_{14}$ (218.3) Ber. C 93.54 H 6.46 Gef. C 93.14 H 6.21}

Thermolyse von 1,2,3,4-Tetrahydro-1-phenyl-1,2,3-methenonaphthalin (35): Die Probenvorbereitung geschah wie bei der Thermolyse von 27. Die Anfangskonzentration ermittelte man anhand des Integrals des Dubletts von $35^{[21 a]}$ bei $\delta=2.62$ im Vergleich zum Integral des Cyclohexen-Signals bei 5.60. Je zwei Proben wurden bei $111.4,119.9,129.2$ und $136.3^{\circ} \mathrm{C}$ umlagert. Der Fortgang der Reaktion wurde durch Integration des Doppeldubletts von 3,7bDihydro-7b-phenyl-2aH-cyclobut [a]inden (36) bei $\delta=5.90$ über 2.2-4.1 Halbwertszeiten beobachtet. Beim Endwert lagen die NMR-spektroskopisch bestimmten Ausbeuten von 36 bei 88-97\%. Zur Isolierung von 36 wurde eine Probe nach der Messung der Kinetik i. Vak. eingeengt. Aus dem Rückstand destillierte 36 bei $70^{\circ} \mathrm{C}(\mathrm{Bad}) / 10^{-3}$ Torr als zähes farbloses $\circlearrowright \mathrm{l}$. - MS $(70 \mathrm{eV})$ : $m / z(\%)=218(100)\left[\mathrm{M}^{+}\right], 217(66), 216(15), 215(34), 203$ (56), 202 (54), 189 (17), 141 (13), 115 (18), 101 (16). - ${ }^{1}$ H-NMR: Tab. 2. - ${ }^{13}$ C-NMR: Tab. 3.

$\mathrm{C}_{17} \mathrm{H}_{14}$ (218.3) Ber. C 93.54 H 6.46 36: Gef. C 93.91 H 6.67 37: Gef. C 93.61 H 6.51

Eine Lösung von 36, die durch Umlagerung von $250 \mathrm{mg}$ (1.15 mmol) 35 erhalten worden war, erhitzte man $3 \mathrm{~h}$ auf $160^{\circ} \mathrm{C}$. Danach waren die ${ }^{1} \mathrm{H}-\mathrm{NMR}$-Signale von 36 verschwunden und nur die von 9-Phenyl-5H-benzocyclohepten (37) beobachtbar. Durch Einengen i. Vak. und Destillation bei $70^{\circ} \mathrm{C}(\mathrm{Bad}) / 10^{-3}$ Torr wurden $230 \mathrm{mg}$ $(92 \%) 37$ als farbloses $\mathrm{Ol}$ isoliert, das in der Vorlage kristallisierte, Schmp. $96-98^{\circ} \mathrm{C}$. $-\mathrm{MS}(70 \mathrm{eV}): m / z(\%)=218(100)\left[\mathrm{M}^{+}\right], 217$ (59), 216 (12), 215 (35), 203 (50), 202 (50), 189 (12), 141 (12), 115 (16), 108 (14), 101 (17), $94(11) .-{ }^{1} \mathrm{H}-\mathrm{NMR}\left(\mathrm{CDCl}_{3}\right): \delta=3.10$ (d, $J_{5,6}=$ $\left.6.5 \mathrm{~Hz} ; 5-\mathrm{H}_{2}\right), 5.90\left(\mathrm{dt}, J_{6.7}=9.6 \mathrm{~Hz} ; 6-\mathrm{H}\right), 6.14\left(\mathrm{dd}, J_{7,8}=5.3 \mathrm{~Hz}\right.$; 7-H), 6.72 (d; 8-H), 7.00-7.50 (m; 1,2,3,4-H), $7.35\left(\mathrm{~s} ; \mathrm{C}_{6} \mathrm{H}_{5}\right) .-{ }^{13} \mathrm{C}$ NMR $\left(\mathrm{CDCl}_{3}\right): \delta=34.8(\mathrm{t} ; \mathrm{C}-5), 125.1,126.2,126.9,127.2,128.6$, 
128.9, 129.3 (jeweils d; C-1,2,3,4,6,7,8, p-C), 128.2, 129.2 (jeweils d; $o, m-C), 137.4,139.0,144.1,145.8$ (jeweils s; C-4a,9,9a, ipso-C).

\section{CAS-Registry-Nummern}

7: 287-13-8 / 9: 4054-38-0 / 11 : 4927-03-1 / 17: 55701-54-7 / 17a: 138089-57-3 / 18: 3725-37-9 / 18a: 138089-58-4 / 19: 3725-38-0 20: 57293-39-7 / 21: 57293-41-1 / 22: 138089-59-5 / 23: 5729340-0 / 24: 138089-60-8 / 25: 56594-63-9 / 26: 138089-61-9 / 27 : 138089-62-0 / 28: 138089-63-1 / 29: 138089-64-2 / 30: 138089-65-3 / 31: 138089-66-4 / 32: 135042-38-5 / 33: 138089-67-5 / 34: 13808968-6 / 35: 138089-69-7 / 36: 138089-70-0 / 37: 138089-71-1 / 3-Phenyltricyclo[4.1.0.0,7 heptan: 138089-72-2 / endo-6-Phenyltricyclo[3.2.0.0.17]heptan: 138089-73-3

I' Aus: M. Christl, Habilitationsschrift, Univ. Würzburg, 1977. ${ }^{[2]}$ Aus: R. Stangl, Dissertation, Univ. Würzburg, 1984.

${ }^{[3]}$ Aus: H. Jelinek-Fink, Dissertation, Univ. Würzburg, 1991.

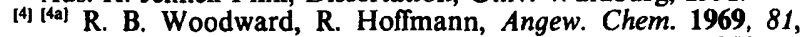
797-896; Angew. Chem. Int. Ed. Engl. 1969, 8, 781-853. ${ }^{[46]}$ M. J. S. Dewar, Angew. Chem. 1971, 83, 859-875; Angew. Chem. Int. Ed. Engl. 1971, 10, 761-776.

[5] $\mathrm{S}$. Hoz in The Chemistry of the Cyclopropyl Group (Hrsg.: S. Patai, Z. Rappoport), Wiley, Chichester, 1987, S. 1121-1192 (Teil 2).

${ }^{16)}$ H. M. Frey, I. D. R. Stevens, Trans. Faraday Soc. 1965, 61, 90-94.

${ }^{7}$ R. Srinivasan, A. A. Levi, I. Haller, J. Phys. Chem. 1965, 69, $1775-1777$.

${ }^{[8]}$ K. B. Wiberg, J. M. Lavanish, J. Am. Chem. Soc. 1966, 88, $5272-5275$.

[9] P. B. Shevlin, M. L. McKee, J. Am. Chem. Soc. 1988, 110, $1666-1671$.

${ }^{[10]}$ G. L. Closs, P. E. Pfeffer, J. Am. Chem. Soc. 1968, 90, $2452-2453$.

${ }^{[11]}$ M. J. S. Dewar, S. Kirschner, J. Am. Chem. Soc. 1975, 97, 2931 -2932; M. J. S. Dewar, ibid. 1984, 106, 209-219.

${ }^{[12]}$ G. M. Lampman, J. C. Aumiller, Org. Synth. 1988, Coll. Vol. 6, 133-136; D. Seebach, R. Hässig, J. Gabriel, Helv. Chim. Acta $1983,66,308-337$.

[13] W. R. Moore, H. R. Ward, R. F. Merritt, J. Am. Chem. Soc. $1961,83,2019-2020$.

${ }^{114]}$ J. Backes, U. H. Brinker in Methoden der organischen Chemie (Houben-Weyl) 4. Aufl., 1989, Bd. E 19b, S. 391-510 (Teil 1)

${ }^{[15]}$ K. B. Wiberg, G. Szeimies, Tetrahedron Lett. 1968, 1235-1239.

${ }^{[16]}$ G. R. Branton, H. M. Frey, D. C. Montague, I. D. R. Stevens, Trans. Faraday Soc. 1966, 62, 659-663.

[17] [17a] M. Christl, U. Heinemann, W. Kristof, J. Am. Chem. Soc. 1975, 97, 2299-2301. - ${ }^{[17 b]}$ Y. Sugihara, T. Sugimura, N. Saito, I. Murata, J. Chem. Soc., Chem. Commun. 1989, 537-539. [i17c] Y. Sugihara, A. Yamato, I. Murata, Tetrahedron Lett. 1981, $22,3257-3260$.

[18] R. Merényi, Z. Janousek, H. G. Viehe in Substituent Effects in Radical Chemistry (Hrsg.: H. G. Viehe, Z. Janousek, R. Merényi), Reidel, Dordrecht, 1986, S. $301-324$.

${ }^{119]}$ D. Hasselmann, K. Loosen, Angew. Chem. 1980, 92, 651-652; Angew. Chem. Int. Ed. Engl. 1980, 19, 634-636.

${ }^{[20]}$ N. J. Turro, V. Ramamurthy, R. M. Pagni, J. A. Butcher, Jr., $J$. Org. Chem. 1977, 42, 92-96.

[21) (21 a) R. Stangl, H. Jelinek-Fink, M. Christl, Chem. Ber. 1992, 125 $479-484$, voranstehend. - $[216]$ H. Jelinek-Fink, M. Christl, E.-M. Peters, K. Peters, H. G. von Schnering, Chem. Ber. 1991 124. $2569-2575$
${ }^{[22]}$ W. R. Roth, H.-W. Lennartz, W. von E. Doering, L. Birladeanu, C. A. Guyton, T. Kitagawa, J. Am. Chem. Soc. 1990, 112, $1722-1732$

[23] [23a] H. H. Freedman, G. A. Doorakian, V. R. Sandel, J. Am Chem. Soc. 1965, 87, 3019-3020. - ${ }^{[23 b]}$ M. Pomerantz, P. H. Hartman, Tetrahedron Lett. 1968, 991 - 993. - ${ }^{[23 c]}$ J. I. Brauman, W. C. Archie, Jr., Tetrahedron 1971, 27, 1275-1280. [23d] W. Cooper, W. D. Walters, J. Am. Chem. Soc. 1958, 80, 4220-4224. - ${ }^{[23 \mathrm{e}]}$ M. A. Battiste, M. E. Burns, Tetrahedron Lett. 1966, 523-530.

[24] P. G. Gassman, T. J. Atkins, J. Am. Chem Soc. 1971, 93, 1042-1043; K. B. Wiberg, H. A. Connon, ibid. 1976, 98, $5411-5412$

[25] [25a] M. Christl, M. Lechner, Angew. Chem. 1975, 87, 815-816; Angew. Chem. Int. Ed. Engl. 1975, 14, 765-766. - ${ }^{[25 b]} \mathrm{M}$. Christl, M. Lechner, Chem. Ber. 1982, 115, 1-13.

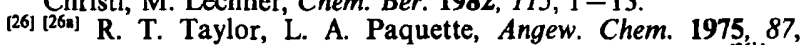
488 -489; Angew. Chem. Int. Ed. Engl. 1975, 14, 496. - [26b] L' A. Paquette, R. T. Taylor, J. Am. Chem. Soc. 1977, 99, $5708-5715$.

[27 W. R. Roth, B. Peltzer, Liebigs Ann. Chem. 1965, 685, 56-74.

${ }^{[28]}$ W. Grimme, W. von E. Doering, Chem. Ber. 1973, 106, $1765-1780$

${ }^{[29]}$ W. von E. Doering, W. R. Roth, Tetrahedron 1963, 19, 715- 737.

${ }^{[30]}$ M. Christl, H. Leininger, P. Kemmer, Chem. Ber. 1984, 117, 2963-2987.

${ }^{[31]}$ K. Fujita, T. Nakamura, K. Matsui, T. Shono, Tetrahedron Lett. 1975, $2441-2444$.

[32] I. Murata, T. Nakazawa, M. Kato, T. Tatsuoka, Y. Sugihara, Tetrahedron Lett. 1975, 1647-1650.

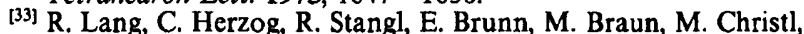
E.-M. Peters, K. Peters, H. G. von Schnering, Chem. Ber. 1990, 123, 1193-1207.

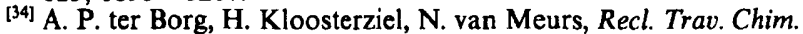
Pays-Bas 1963, 82, 717-740.

${ }^{[35]}$ M. J. S. Dewar, S. Kirschner, J. Am. Chem. Soc. 1974, 96, $6809-6810$

[36] [36a] G. Fraenkel, J. M. Geckle, J. Am. Chem. Soc. 1980, 102, 2869-2880. - ${ }^{[366]}$ H. O. Kalinowski, S. Berger, S. Braun, ${ }^{13} \mathrm{C}$ NMR-Spektroskopie, Thieme, Stuttgart, 1984.

[37] [37a] D. P. G. Hamon, Aust. J. Chem. 1974, 27, 153-161. - ${ }^{\text {[37b] }}$ D. P. G. Hamon, Aust. J. Chem. 1975, 28, 2641 - 2648, - [37c] J. C. Martin, J. E. Schultz, J. W. Timberlake, Tetrahedron Lett. $1967,4629-4634$.

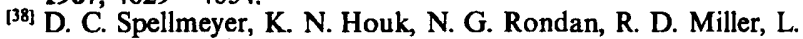
Franz, G. N. Fickes, J. Am. Chem. Soc. 1989, 111, 5356-5367.

${ }^{[39]}$ Dieser Mittelwert ergibt sich durch Vergleich der Hydrierwärmen von Styrol und 1-Buten sowie von $(E)$-Stilben und $(E)-3-$ Hexen: J. B. Pedley, R.D. Naylor, S. P. Kirby, Thermochemical Data of Organic Compounds, 2nd Ed., Chapman and Hall, London, 1986

[40] [400], Y. Sugihara, T. Sugimura, I. Murata, J. Am. Chem. Soc. 1984, 106, $7268-7269$ - ${ }^{[40 \mathrm{~b}]}$ Y. Sugihara, S. Wakabayashi, N. Saito, I. Murata, J. Am. Chem. Soc. 1986, 108, 2773-2775. ${ }_{[40 c]}$ N. J. Turro, C. A. Renner, T. J. Katz, K. B. Wiberg, H. A. Connon, Tetrahedron Lett. 1976, 4133-4136; M. J. S. Dewar, S. Kirschner, J. Am. Chem. Soc. 1975, 97, 2932-2933.

[41] [4ia] P. G. Gassman, G. D. Richmond, J. Am. Chem. Soc. 1970 92, 2090-2096. - ${ }_{1416 \text { M }}$ M. Christl, G. Freitag, G. Brüntrup, Chem. Ber. 1978, 111, 2307-2319.

[42] [42a] W. Metzner W. Hartmann, Chem. Ber. 1968, 101, $4099-4105$. - $^{[426]}$ D. Wendisch, W. Metzner, Chem. Ber. 1968, $101,4106-4122$.

[360/91] 